\title{
Using the Electroretinogram to Understand How Intraocular Pressure Elevation Affects the Rat Retina
}

\author{
Bang V. Bui, ${ }^{1}$ Zheng He, ${ }^{1}$ Algis J. Vingrys, ${ }^{1}$ Christine T. O. Nguyen, ${ }^{1}$ \\ Vickie H. Y. Wong, ${ }^{1}$ and Brad Fortune ${ }^{2}$ \\ ${ }^{1}$ Department of Optometry and Vision Sciences, University of Melbourne, Parkville, VIC 3010, Australia \\ ${ }^{2}$ Devers Eye Institute and Legacy Research Institute, Legacy Health, Portland, OR 97232, USA
}

Correspondence should be addressed to Bang V. Bui; bvb@unimelb.edu.au

Received 11 September 2012; Accepted 24 October 2012

Academic Editor: Shigeki Machida

Copyright (C) 2013 Bang V. Bui et al. This is an open access article distributed under the Creative Commons Attribution License, which permits unrestricted use, distribution, and reproduction in any medium, provided the original work is properly cited.

\begin{abstract}
Intraocular pressure (IOP) elevation is a key risk factor for glaucoma. Our understanding of the effect that IOP elevation has on the eye has been greatly enhanced by the application of the electroretinogram (ERG). In this paper, we describe how the ERG in the rodent eye is affected by changes in IOP magnitude, duration, and number of spikes. We consider how the variables of blood pressure and age can modify the effect of IOP elevation on the ERG. Finally, we contrast the effects that acute and chronic IOP elevation can have on the rodent ERG.
\end{abstract}

\section{Introduction}

The retina is unique in its specialisation for the capture of light and transduction into an electrical signal. Effective phototransduction and signal transmission involves many processes that are energetically expensive, including the maintenance of transmembrane ionic gradients, signal amplification, and neurotransmission. Not surprisingly, the retina is the most metabolically demanding of all the body's tissues $[1,2]$. Given such a metabolic burden the neural retina may be uniquely susceptible to metabolic stress and disease.

Glaucoma is the second leading cause of blindness in Australia [3] and worldwide [4], with intraocular pressure (IOP) being a key risk factor [5]. IOP reduction is an effective treatment regardless of the type of glaucoma [6, 7]. However, glaucomatous neuropathy can occur in the presence or absence of a detectable IOP elevation. The role that IOPrelated stress may have in the development and progression of glaucoma is not fully understood.

The retinal response to a flash of light is termed the electroretinogram (or ERG) and is a commonly used assay of retinal function. The ERG sums the field potentials of various retinal cells following light stimulation [8]. The ERG is noninvasive, easy to measure, and highly reproducible $[9,10]$ and is thus ideally suited to assess the retina as a whole. Decomposition of the overall voltage envelope into its constituent parts allows the underlying cell classes to be considered with some level of specificity [8]. a-wave and bwave features of the bright flash ERG are excellent indicators of photoreceptoral and bipolar cell activity [11]. It needs to be mentioned that although the scotopic rat a-wave primarily reflects photoreceptor activation it also has inputs from second-order neurons that modulate the waveform beyond $14 \mathrm{~ms}$ [12]. Responses collected for very dim stimulus flash energies-equate to behavioral light detection thresholds [13] - are known as the scotopic threshold response (STR), which largely reflects the integrity of retinal ganglion cells in rodents $[14,15]$. The high-frequency wavelets residing on the leading edge of the b-wave (oscillatory potentials, OPs) are a useful indicator of inner retinal activity including that of amacrine cells [16]. Thus in a relatively short recording period, information regarding the health of broad retinal cell classes can be collected.

A more extensive discussion of the application of the ERG on rodents can be found elsewhere [17]. This paper describes the application of the ERG to study the effects of metabolic inhibition and IOP-related stress on the retina. The paper focuses on the effect of stress on the rat retina, an increasingly utilised model of retinal diseases, including glaucoma. 


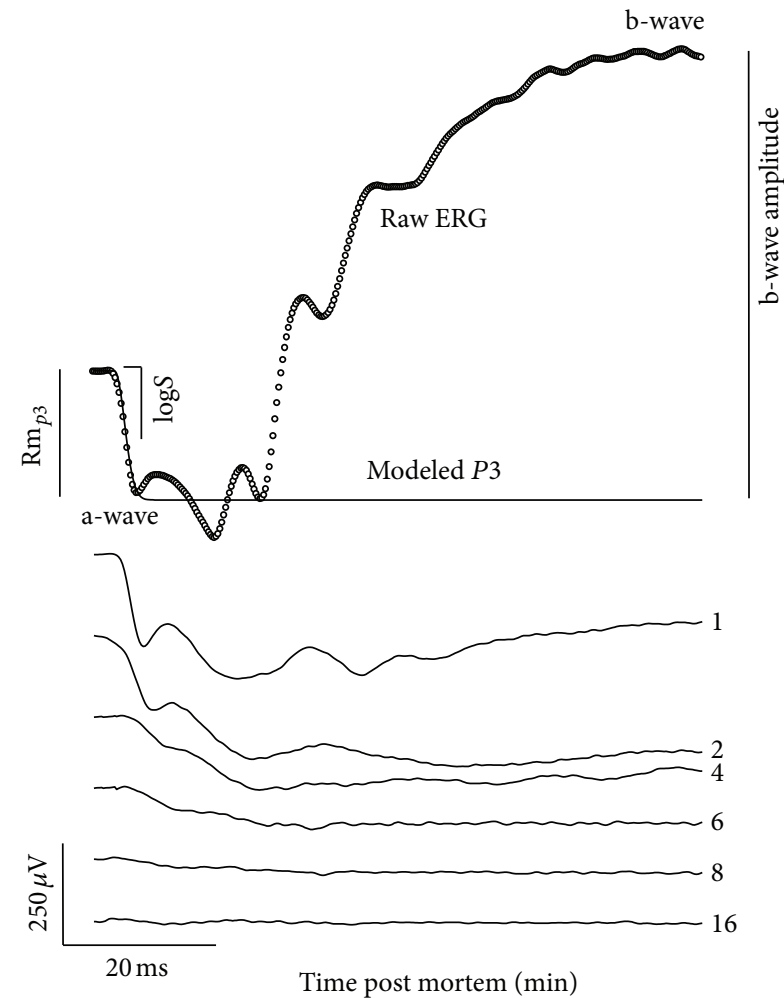

(a)

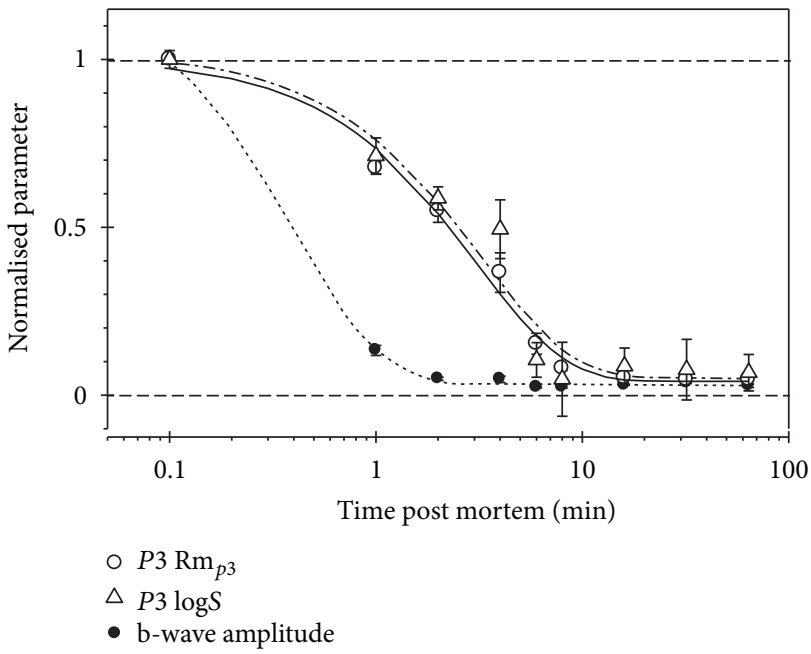

(b)

FIGURE 1: Retinal function is rapidly lost in the rat retina following acute complete inhibition of metabolite supply. (a) The leading edge of the raw response (a-wave, unfilled circles) was modeled using a phototransduction model ( $P 3$, thick line). (b) Postreceptoral b-wave (filled circles) is more susceptible than phototransduction amplitude $\left(\mathrm{Rm}_{P 3}\right.$, unfilled circles) and sensitivity (log $S$, unfilled triangles). Reprinted from [24] with permission. The Association for Research in Vision and Ophthalmology remains the copyright holder.

\section{Metabolic Stress and the ERG}

Previous studies have shown that the ERG is exquisitely sensitive to metabolic stress. In general such studies show that the ERG is rapidly lost when normal metabolic substrate supply is interrupted, as occurs following hypoglycaemia [18], hypoxia $[19,20]$, and acute ischemia [21] most commonly achieved via interruption of the blood supply [22, 23].

The rate at which retinal function declines after complete impairment of substrate supply can be interpreted as a measure of endogenous substrate availability (e.g., remaining adenosine triphosphate, oxygen, glucose, and glycogen). Figure 1 shows that the dark-adapted photoreceptoral a-wave in Sprague-Dawley rats continues to be measurable for up to 10 minutes following complete ischemia [24].

The photoresponse can be described using a mathematical model based on known biochemical cascade of phototransduction to provide a measure of the saturated dark current $\left(\mathrm{Rm}_{P 3}\right)$ and phototransduction sensitivity $(\log S)$ or amplification $[25,26]$. Figure $1(\mathrm{~b})$ shows that $\mathrm{Rm}_{P 3}$ and $\log S$ decay at a similar rate following hypoxemic insult, suggesting that phototransduction gain depends on the same energy producing mechanisms that maintain the $\mathrm{Na}^{+} / \mathrm{K}^{+}$ATPases involved in restoring the dark current. It is evident from Figure 1 that the ERG b-wave is diminished within 1 to 2 minutes following the onset of complete ischemia. These data are consistent with previous findings that the inner retina is more sensitive to metabolic stress than is the outer retina.

The rate of ERG decline following metabolic challenge is constrained by the availability of remnant metabolic substrates including those that can be mobilised from endogenous stores. This explains the greater susceptibility of the b-wave to ischemic insult compared to the a-wave, as the photoreceptor sequesters its neurotransmitter (glutamate) to sustain metabolism at the expense of synaptic transmission [24]. Thus conditions that reduce the use of such substrates should delay the loss of function as previously suggested $[27,28]$. One manner in which metabolic consumption can be reduced is via light adaptation, which has been shown to attenuate oxygen [29] and glucose consumption [30] particularly at photoreceptor inner segments [31].

Figure 2 shows that light-adapted ERGs in rats have a smaller a-wave and slower b-wave compared with humans [32] and nonhuman primates [33]. A smaller a-wave is consistent with fewer cones in rat retinae [34]. Similar to scotopic [35], and photopic [33] a-wave from nonhuman 


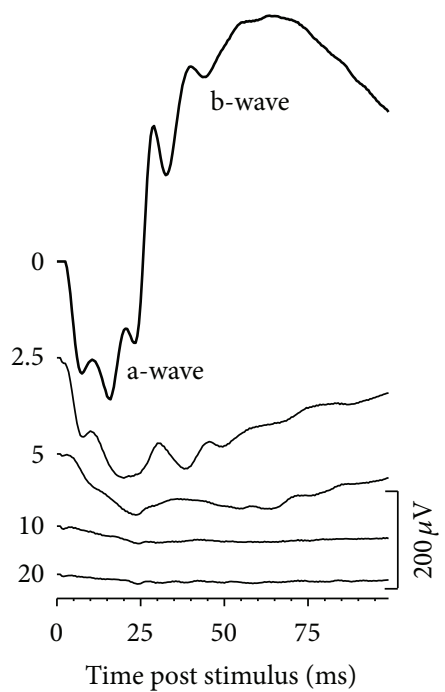

(a)
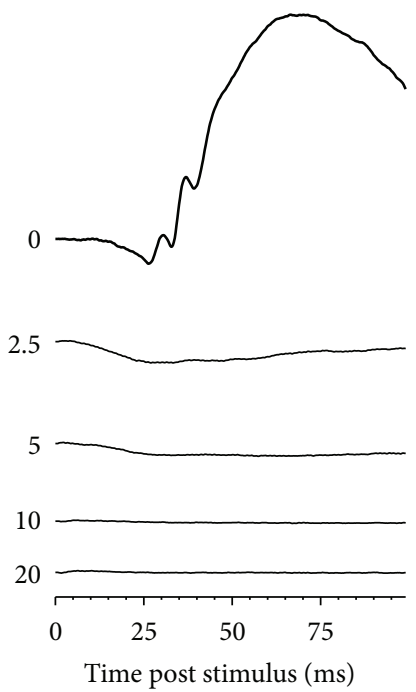

(b)

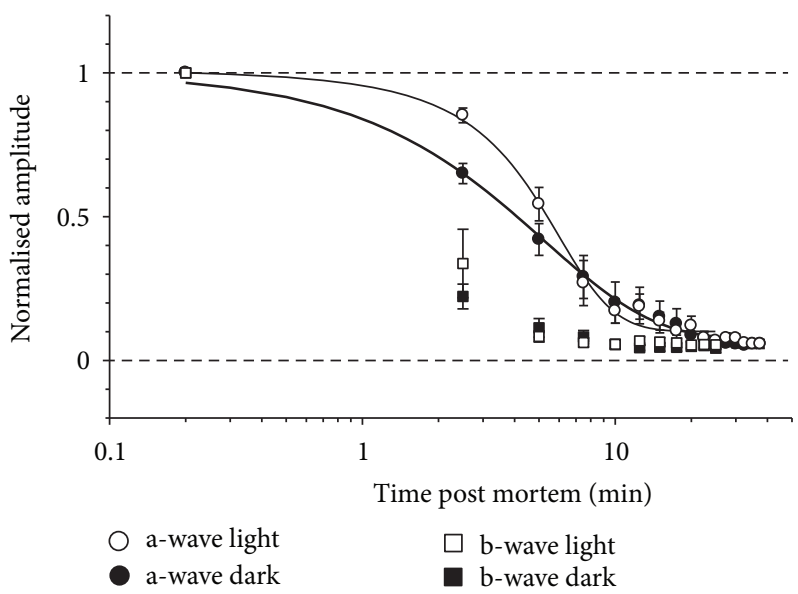

(c)

FIGURE 2: Light adapting the retina results in relatively slower decay of a-wave amplitudes at 2.5 and 5 minutes following ischemic insult. ERG loss under dark- (a) and light-adapted conditions (b). Group data are shown in (c). Reprinted from [24] with permission. The Association for Research in Vision and Ophthalmology remains the copyright holder.

primates, the scotopic a-wave in rats [12] also contains contributions from hyperpolarising bipolar cells. Despite a smaller a-wave, the light-adapted b-wave in rats is almost $60 \%$ the size of its dark-adapted counterpart, consistent with the relatively large number of cone bipolar cells in the rat retina [34]. In comparison to the dark-adapted condition, the lightadapted a-wave was relatively less attenuated at 2.5 and 5 minutes after ischemic injury (Figures 2(b) and 2(c)).

Our findings are consistent with previous reports that rod responses show greater susceptibility to hypoglycemia than light-adapted cone responses [36, 37]. Whilst both rods and cone may benefit from reduced metabolic demand associated with light adaptation, cones may inherently be less susceptible. For example, cones store glycogen, whereas rods lack glycogen and the rate-limiting glycogenolytic enzyme, glycogen phosphorylase $[38,39]$.

\section{IOP Elevation and the ERG}

Metabolic inhibition can also be induced by elevation of intraocular pressure above the mean arterial blood pressure [20]. Whilst this approach shares similarities with occlusive models of ischemia, IOP elevation also increases mechanical load on retinal tissues $[22,23]$.

The balance between the production and outflow of aqueous humor determines intraocular pressure. This nutritive fluid supports the metabolism of most avascular tissues of the eye. It is actively secreted by the epithelium of the ciliary body into the posterior chamber. From the posterior chamber aqueous moves down an osmotic gradient through the pupil to enter the anterior chamber. The primary outflow pathway is through the trabecular meshwork, with a small percentage (10\%) exiting via the uveoscleral route. Thus, any resistance 


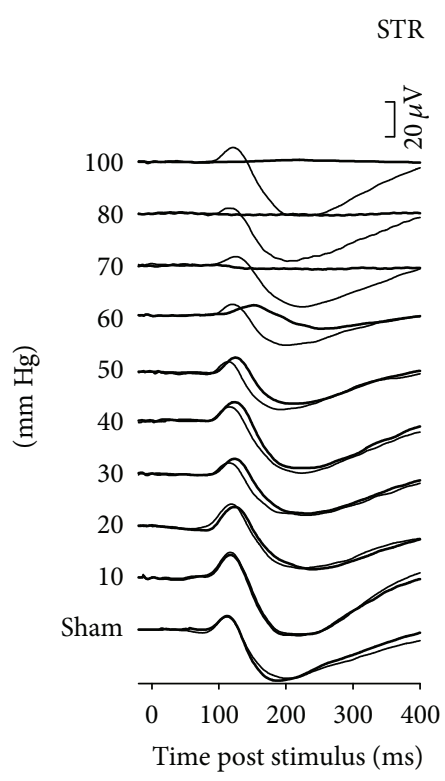

(a)

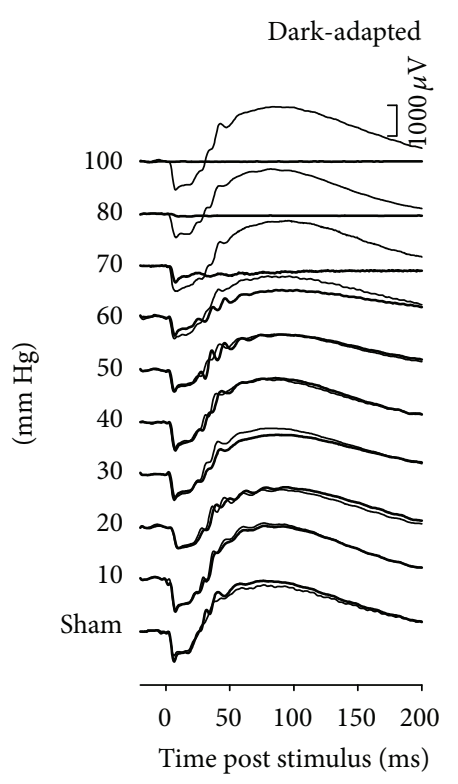

(b)

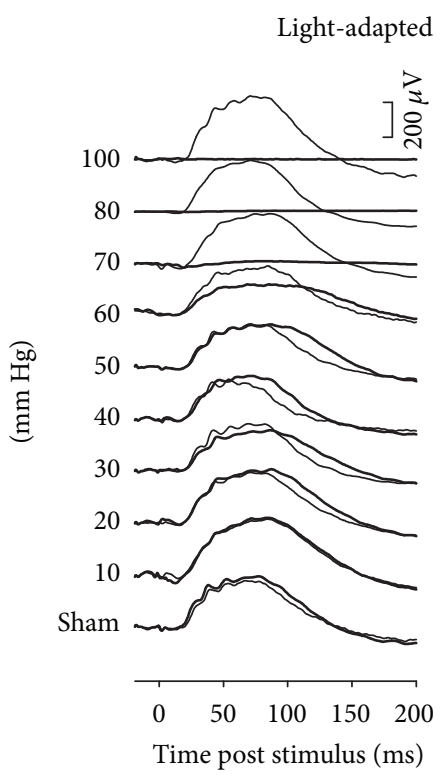

(c)

FIGURE 3: Rat ERG response to various levels of acute IOP elevation. (a) Scotopic threshold response (STR, $-5.55 \log c d s \mathrm{~m}^{-2}$ ). (b) Scotopic bright flash ERG $\left(2.22 \log \mathrm{cd} \mathrm{s} \mathrm{m}^{-2}\right)$. (c) Photopic ERG $\left(2.72 \log \mathrm{cd} \mathrm{s} \mathrm{\textrm {m } ^ { - 2 }}\right.$ on a steady white background of $\left.150 \mathrm{~cd} \cdot \mathrm{m}^{-2}\right)$. ERG responses for fellow control eyes are shown by the thin traces in each column. Reprinted from [46] with permission. The Association for Research in Vision and Ophthalmology remains the copyright holder.

in the outflow (e.g., blockage in trabecular meshwork) or a change in the anatomic relationship between the pupillary margin and the lens will result in IOP elevation [40-42].

Whilst it is clear that elevated IOP is an important risk factor for glaucoma [43], the elevated IOP hypothesis fails to account for the fact that many glaucoma patients (20-30\%) have normal pressure [5, 44]. In addition, the disease can progress in $20 \%$ of eyes even though IOP has been substantially ( $\geq 30 \%$ ) lowered [45], whereas over half of untreated individuals with ocular hypertension do not show visual field progression $[44,45]$. Such apparent clinical paradoxes may arise from an incomplete understanding of how change in IOP affects visual function and neuronal integrity. Thus a deeper understanding of how IOP influences the integrity of the retina is crucial to understanding the pathophysiology of glaucoma. We have considered these issues with an acute IOP insult as will be described next.

Figure 3 shows that acute IOP elevation produces a doserelated decline in the ERG. During IOP elevation reductions in ERG amplitude are not seen until IOP is elevated to $60 \mathrm{mmHg}$ or more. For pressures beyond $60 \mathrm{mmHg}$ the ERG shows progressively greater attenuation and is abolished at $100 \mathrm{mmHg}$.

3.1. Which Component of the ERG Is Most Sensitive to IOP Elevation? A number of studies have compared the effect of IOP elevation on ERG components whose cellular origins have been attributed to outer, middle, and inner retina. These studies are in agreement that the outer retina is less sensitive to IOP elevation than is the inner retina. Foulds and Johnson [47] reported that the a-wave recovered faster than the b-wave following IOP elevation. Furthermore, comparison between outer retinal and ganglion cell function achieved by simultaneously measuring the flash ERG (b-wave) and pattern ERG, respectively, are consistent with greater inner retinal sensitivity to IOP elevation. Specifically, both chronic IOP elevation in monkeys [48] and acute IOP elevation in cats [49] and rabbits [50] resulted in decreased pattern ERG amplitudes, whereas the ERG b-wave remained unchanged.

As shown in Figure 3 and summarised in Figure 4 the nSTR and photopic OPs of full-field ERG are most sensitive to acutely elevated IOP. The photopic b-wave was the third most sensitive component by amplitude analysis, as it was reduced by $50 \%$ for an IOP level of $\sim 60 \mathrm{mmHg}$. We have previously shown that the STR, photopic b-wave, and photopic OPs were the most sensitive components of the rat full-field ERG to removal of intact ganglion cell function, via optic nerve transection and intravitreal injection of tetrodotoxin [14]. The finding that acute IOP elevation has the largest impact on all of these full-field ERG components suggests that the elements most sensitive to acutely elevated IOP in the rat ERG arise from the inner retina, and in particular intact ganglion cell function (also see Figure 6). This pattern of deficit is consistent with the idea that the large volume and high flow of the choriocapillaris provide a robust buffer against IOP elevation in the rat thereby preserving outer retinal components. The resistance of the rat choroidal perfusion to IOP elevation has been recently demonstrated using optical coherence tomography/optical microangiography [51].

3.2. How Quickly Does the ERG Recover from Stress? Central to the capacity of the eye to resist injury is its ability to recover from stress. In response to complete ischemia induced by IOP elevation (100 mmHg for 60 minutes) our group, as well 


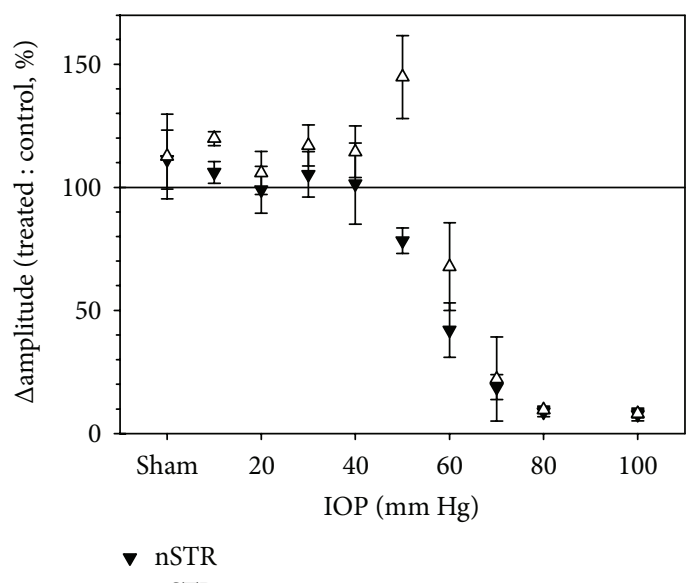

(a)

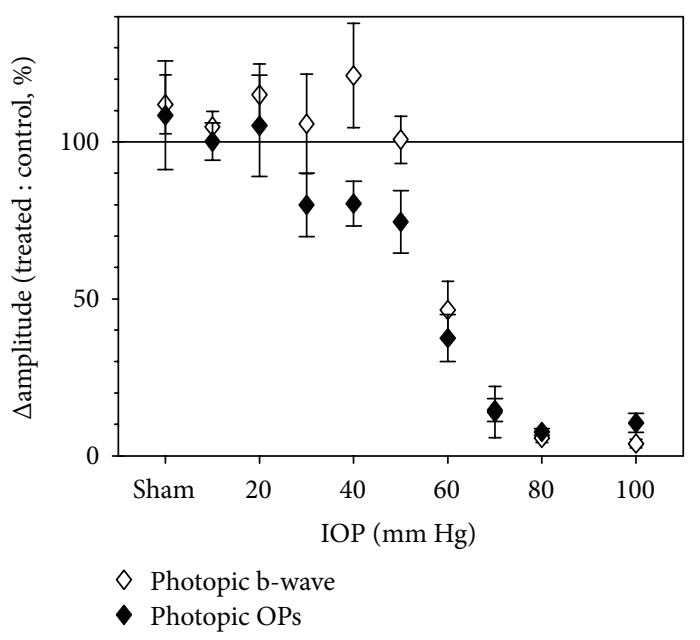

(c)

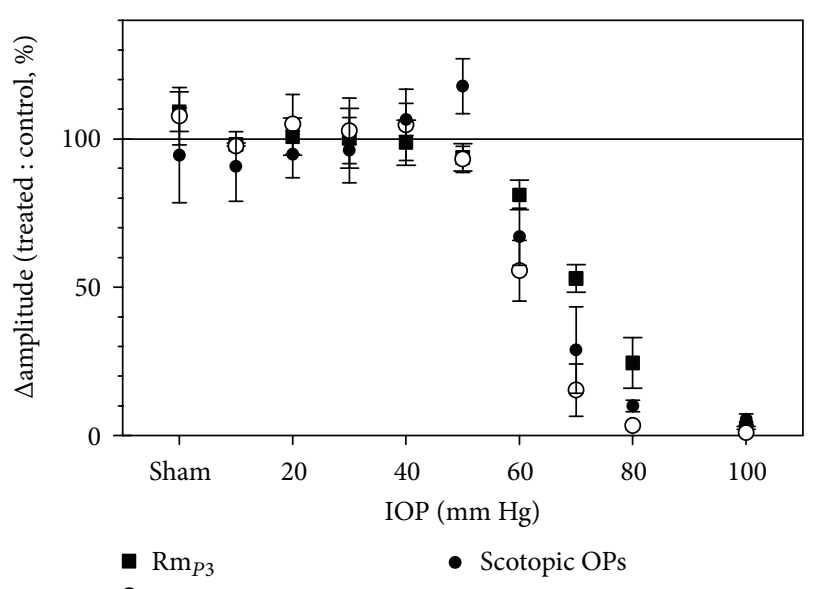

(b)

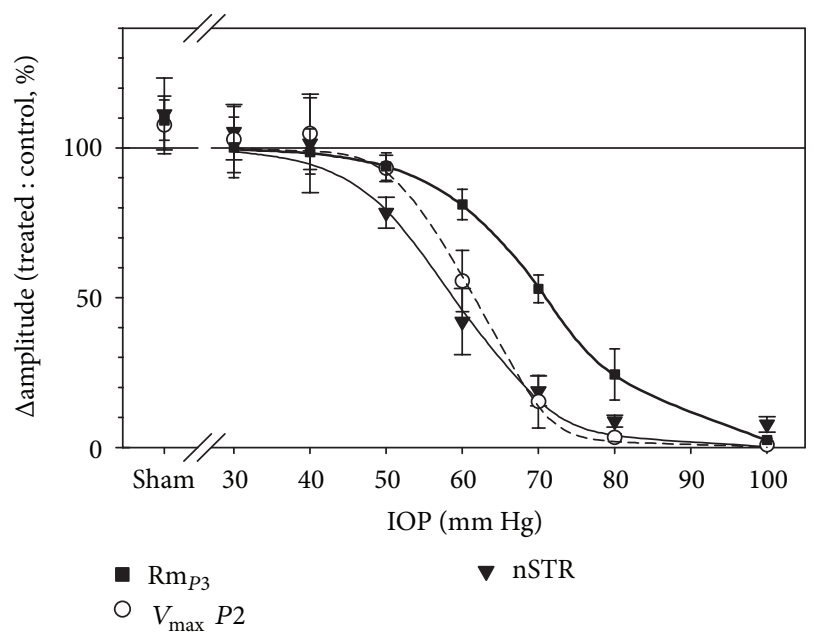

(d)

FIGURE 4: Relative change in ERG amplitude versus IOP (mean \pm SEM). (a) Positive STR (pSTR, unfilled triangles) and negative STR (nSTR, filled triangles). (b) Photoreceptoral amplitude $\left(\mathrm{Rm}_{P 3}\right.$, filled squares), $P 2$ amplitude ( $V_{\max }$, open circles), and scotopic OP amplitude (filled circles). (c) Photopic b-wave amplitude (open diamonds) and photopic OP amplitude (filled diamonds). (d) nSTR (filled triangles, thin solid curve), $P 2 V_{\max }$ (open circles, dashed curve), and photoreceptoral $\mathrm{Rm}_{P 3}$ (filled squares, bold curve) are replotted together with their corresponding best-fit cumulative normal functions for comparison. Reprinted from [46] with permission. The Association for Research in Vision and Ophthalmology remains the copyright holder.

as others, have shown that the ERG does not recover fully. Indeed, there is often a severe reduction of the ERG b-wave a week after the insult $[22,23,52]$. As was the case during IOP elevation ERG components arising from the outer retina show better recovery than do those generated by the inner retina. It is of interest that ischemia-reperfusion produces a relatively greater effect on the cone ERG [53] isolated using a paired or twin flash paradigm [54-56]. Why reperfusion injury should affect the cone ERG more than the rod ERG, when the converse was the case during ischemic stress (Figure 2) is not clear.

In contrast, lower levels of IOP elevation produce reversible ERG losses, as illustrated in Figure 5. Indeed, once IOP had been returned back to baseline the ERG rapidly recovers. Overall there was more severe dysfunction during
IOP elevation at $70 \mathrm{mmHg}$ compared with $50 \mathrm{mmHg}$. For IOP elevation to $50 \mathrm{mmHg}$ (42 minutes) the ON-bipolar cell $P 2$ had largely recovered within a minute after restoration of normal IOP. The inner retinal nSTR took more than 45 minutes to show a similar relative level of recovery. Both bright flash (a-wave and b-wave, Figure 5(a)) and STR responses (Figure 5(b)) take longer to recover in the case of more severe ERG attenuation ( $70 \mathrm{mmHg}$ ).

The above data show that the severity of injury, as indicated by the magnitude of ERG amplitude attenuation, determines how long it takes to recover from stress. Figure 6 confirms that the component of the ERG that recovers fastest is that which was least affected, the photoreceptoral P3. The ON-bipolar cell $P 2$ is the next to recover and finally the inner retinal nSTR. Thus the sensitivity of the inner retina to IOP 


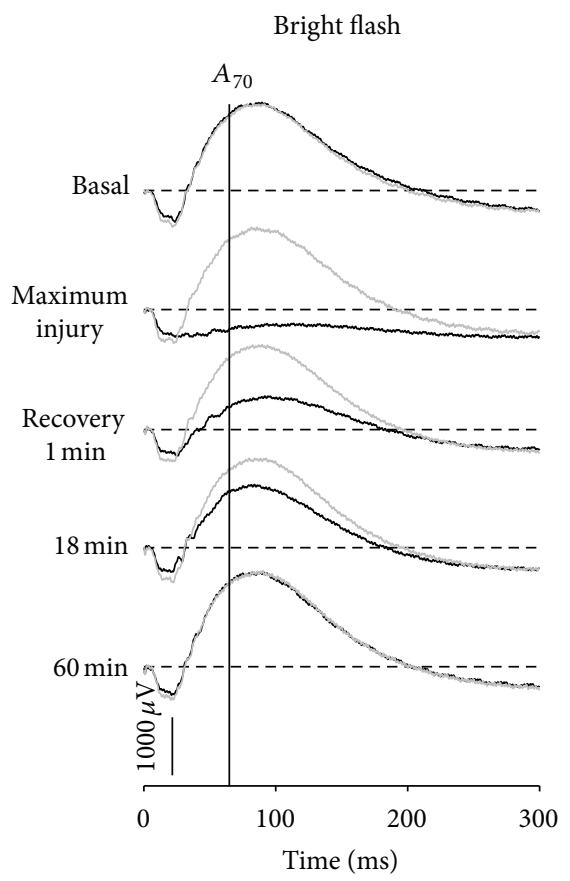

(a)

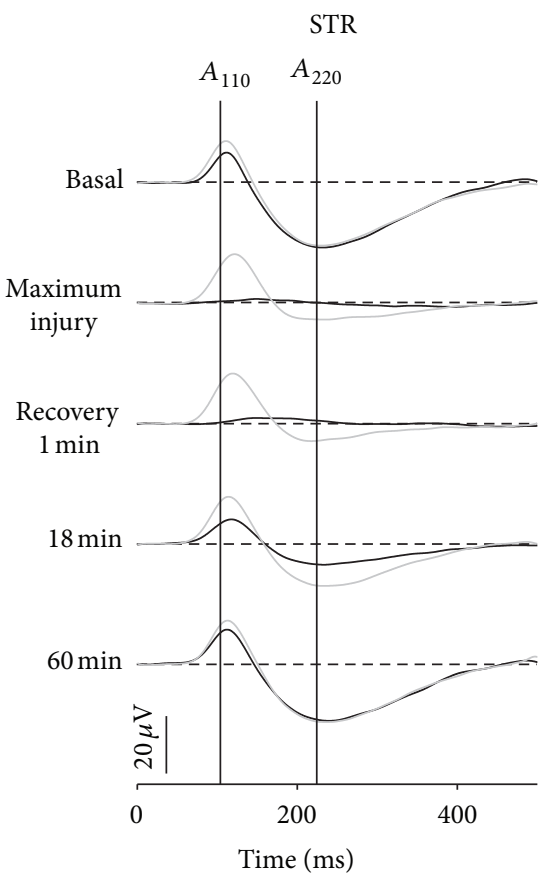

(b)

FIGURE 5: Recovery of bright flash ((a) $\left.1.0 \mathrm{log} \mathrm{cd} \cdot \mathrm{s} \cdot \mathrm{m}^{-2}\right)$ and STR waveforms ((b) $\left.-4.8 \mathrm{log} \mathrm{cd} \cdot \mathrm{s} \cdot \mathrm{m}^{-2}\right)$ from IOP elevation to $50 \mathrm{mmHg}$ for $42 \mathrm{~min}$ (greyed traces) and $70 \mathrm{mmHg}$ for $30 \mathrm{~min}$ (thick traces). Reprinted from [57] with permission. The Association for Research in Vision and Ophthalmology remains the copyright holder.

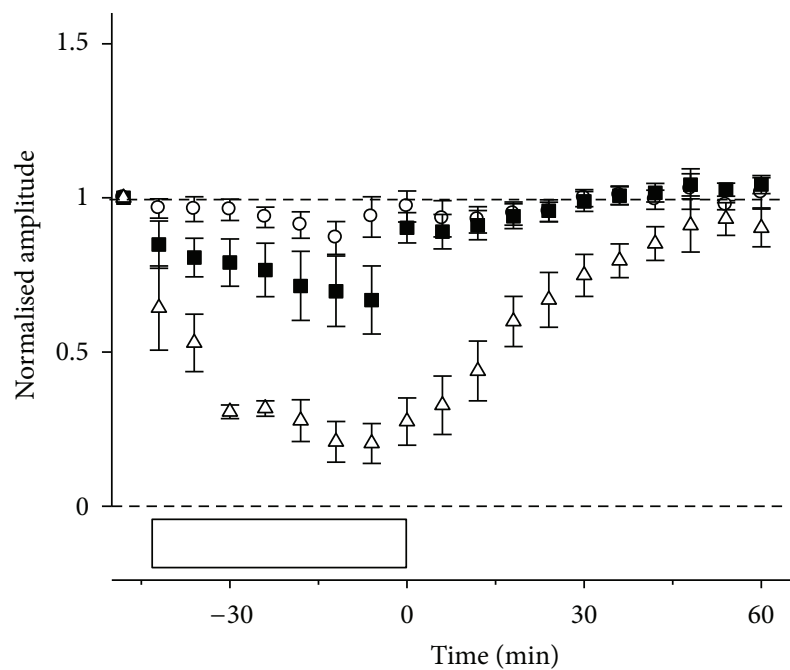

FIGURE 6: Relative amplitude of ERG components (mean \pm SEM) during and after IOP elevation to $50 \mathrm{mmHg}$ for $42 \mathrm{~min}$. $P 3$ (circles), P2 (squares), and nSTR (triangles) amplitudes are compared. Horizontal reference lines indicate baseline and zero levels. Open boxes show the duration of IOP elevation. Reprinted from [57] with permission. The Association for Research in Vision and Ophthalmology remains the copyright holder.

elevation is expressed in two ways, first a larger magnitude of ERG reduction and second a delay in recovery. Both of these are useful indices of the retinal capacity to overcome stress.
Several factors may account for the greater sensitivity of the inner retina to IOP elevation. IOP elevation has the potential to produce both vascular and mechanical stress at the level of the retina and particularly the optic nerve head. The presence of optic nerve stress would have a greater impact on ganglion cell function compared with ERG components arising from middle and outer retinal layers. Moreover, a number of studies have shown that IOP-related stress (force/cross-sectional area) is magnified at the optic nerve head in nonhuman primates [58, 59] and rats [51, 60]. An altered pressure gradient across this tissue makes ganglion cell axons and small blood vessels traversing the optic nerve head particularly susceptible to IOP-related stress.

3.3. Which Is More Detrimental to the ERG, the Magnitude or the Duration of IOP Elevation? Whilst it is clear that the magnitude of IOP elevation has a profound effect on the ERG, there is growing recognition that the duration of IOP elevation is also important. The concept of the total integral of injury (pressure $\times$ duration) accounts for substantial variation in the degree of functional and histological damage in various models of chronic IOP elevation [6165]. We have shown that the grade of optic nerve injury was more closely related to the mean IOP than the peak IOP in rats with chronic IOP elevation [66]. Another way to try and disentangle the effects of IOP magnitude from duration is to consider the effect of IOP elevations of different magnitude but of equivalent IOP integral. Figure 7 plots the time taken for $50 \%$ ERG recovery as a function of IOP 


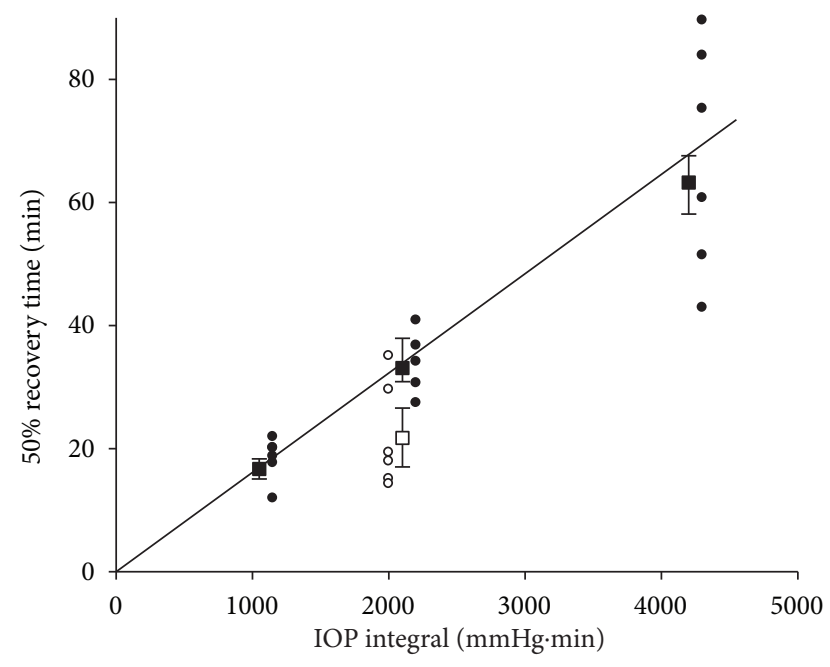

FigURE 7: Relationship between the $50 \%$ recovery time (mins) for nSTR amplitude as a function of IOP time integral for elevation to 70 (filled symbol) and $50 \mathrm{mmHg}$ (unfilled symbols). Individual animals are shown, and squares indicate bootstrapped mean for group data; error bars, $2.5 \%$ and $97.5 \%$ bootstrap confidence limit. Reprinted from [57] with permission. The Association for Research in Vision and Ophthalmology remains the copyright holder.

integral (mmHg.min). The filled symbols show that at an IOP of $70 \mathrm{mmHg}$ the time taken for recovery is progressively prolonged as the integral increases. Also plotted on the figure is an IOP elevation to $50 \mathrm{mmHg}$ for a longer time ( 42 minutes) but which produces the same integral as a $70 \mathrm{mmHg}$ IOP spike of 30 minutes duration $(2100 \mathrm{mmHg} \cdot \mathrm{min})$. It is clear that for IOP spikes having equivalent integrals, (as given by IOP magnitude $*$ time) the higher IOP magnitude produces a greater delay in the rate of recovery.

\subsection{Are Repeated IOP Elevations Worse Than a Single Event?} Drance [67] proposed that undetected IOP fluctuations occurring outside of office hours may be responsible for, or contribute to, glaucoma progression. It is well known that IOP has a circadian rhythm in both normal subjects and glaucoma patients $[68,69]$. Peak IOP often occurs during the night or towards the early morning $[69,70]$ in various species, including humans [69], rabbits [71], rats [72], and mice [73]. Saccà et al. [74] report that IOP fluctuations are greater in glaucoma patients compared with age matched controls. A number of studies have suggested that greater IOP fluctuation increases the risk of visual field progression $[75,76]$. Others have failed to find such an association [77].

We considered this issue by evaluating recovery of the ERG following IOP insults of fixed magnitude and duration but varying the number of insults. Figure 8 shows the time taken for $50 \%$ amplitude recovery expressed as a percentage relative to a single IOP insult. Figure 8(a) shows that recovery of the rat ERG after multiple IOP insults becomes slower as the number of insults increases. The exacerbation of functional deficit is more pronounced for the nSTR compared with the $P 2$, again highlighting the sensitivity of the inner retina.
That recovery is progressively prolonged as the number of insults increased is expected as the IOP integral also increases, and we have shown in Figure 7 that IOP integral has a linear relationship with recovery time. A more appropriate way to consider the importance of repeated IOP insults is to match the insults for their total duration (IOP $*$ time integral). That is, will a single spike of 60 minutes produce the same insult as 4 spikes each of 15 minutes? Figure $8(\mathrm{~b})$ shows that a prolonged single 60 minute insult produces more delay in ERG recovery when compared with two 30-minute and four 15-minute insults. Figure 8 (b) also shows that the benefits are most marked for the bipolar cell-derived $P 2$ and not the ganglion cell-derived STR. Figure 8 provides evidence that number of insults is particularly detrimental to retinal ganglion cell function, which does not benefit from shorter durations in IOP elevation.

This leads to the interesting observation that the time taken for nSTR recovery was similar, regardless of the number of insults, as long as the integral was kept constant. In contrast the bipolar cell $P 2$ recovered faster when the insult was divided into multiple short-duration episodes. Taken together, the data indicates that compared to the middle retina, the inner retina does not cope as well with repeated IOP insults.

\subsection{What Underlies the Loss of the ERG Occurring during IOP} Elevation? Elevated IOP is thought to cause retinal damage by mechanical and vascular mechanisms. Vascular injury is likely to arise from direct compression of blood vessels in the optic nerve head and retina. When IOP elevation reduces ocular perfusion pressure (OPP) beyond the capacity for autoregulation, ocular blood flow will be reduced thus producing retinal dysfunction. Consistent with this idea, Figure 9 shows that the ERG amplitude and ocular blood flow decrease as IOP increases.

More interestingly these data show that, for any given IOP, blood flow was relatively more affected than retinal function. These data suggest that some other factor helps to sustain function despite relative ischemia. Thus the ERG does not require $100 \%$ blood flow to remain within normal limits. This outcome suggests that there is range of blood flow that is adequate to supply the energetic needs of the ERG. Previous studies have shown that oxygen rather than blood flow is critical for the health of the retina [20, 79-81] and optic nerve [82]. One mechanism that may help to maintain tissue oxygenation in the presence of IOP-induced reductions in blood flow is to increase oxygen extraction from residual arterial blood during mild ischemia. Data from human cerebral [83-85] and muscular $[86,87]$ circulation consistently show that oxygen extraction increases with decreased blood flow. Tornquist and Alm [88] have shown in porcine retina an increased difference in arterial venous oxygen tension during reduced ocular perfusion, consistent with increased oxygen extraction under these conditions. Whether changes in oxygen extraction occur in chronic disease such as glaucoma requires further study. 


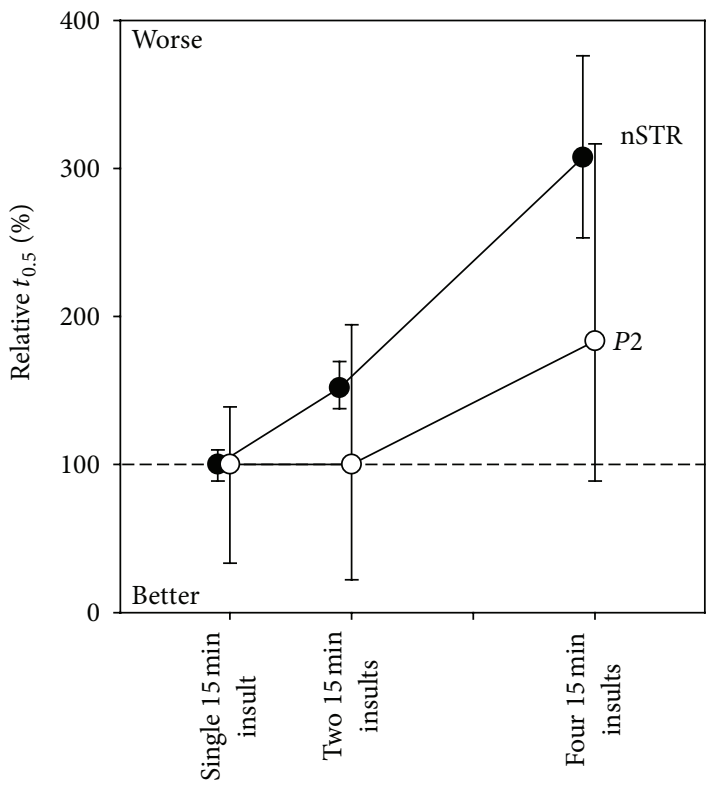

(a) Single versus multiple insults

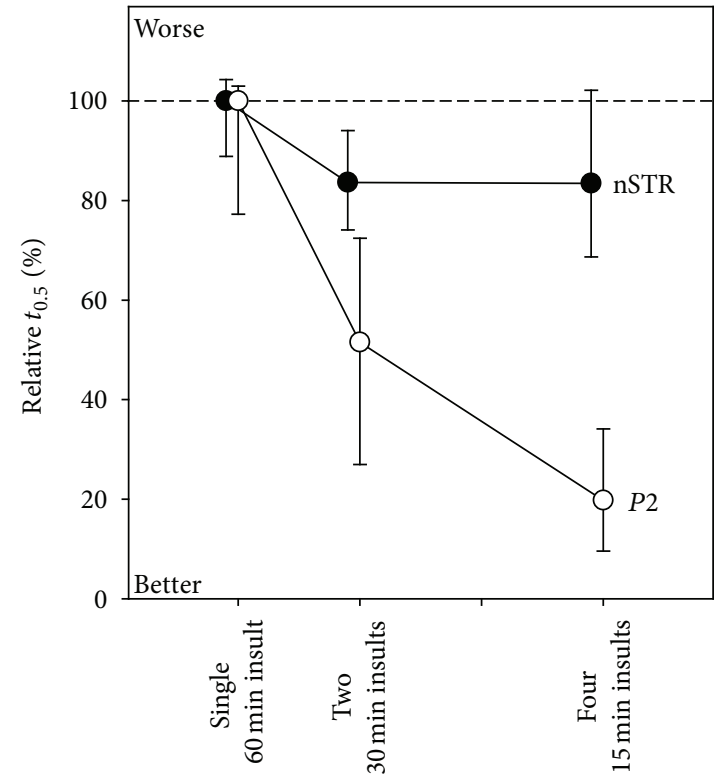

(b) Constant versus varying insult

FIGURE 8: Effect of repeated IOP elevation on ERG recovery. The time taken for $50 \%\left(t_{0.5}\right)$ recovery following repeated IOP insults is expressed relative to a single IOP insult ( $\pm 95 \% \mathrm{CI})$ for ON-bipolar cell b-wave ( $P 2$, unfilled symbols) and inner retinal responses (nSTR, filled symbols). (a) Comparison of single and multiple insults at the same IOP. (b) Comparison of single and multiple insults of equal integral. Reprinted from [78] with permission. The Association for Research in Vision and Ophthalmology remains the copyright holder.

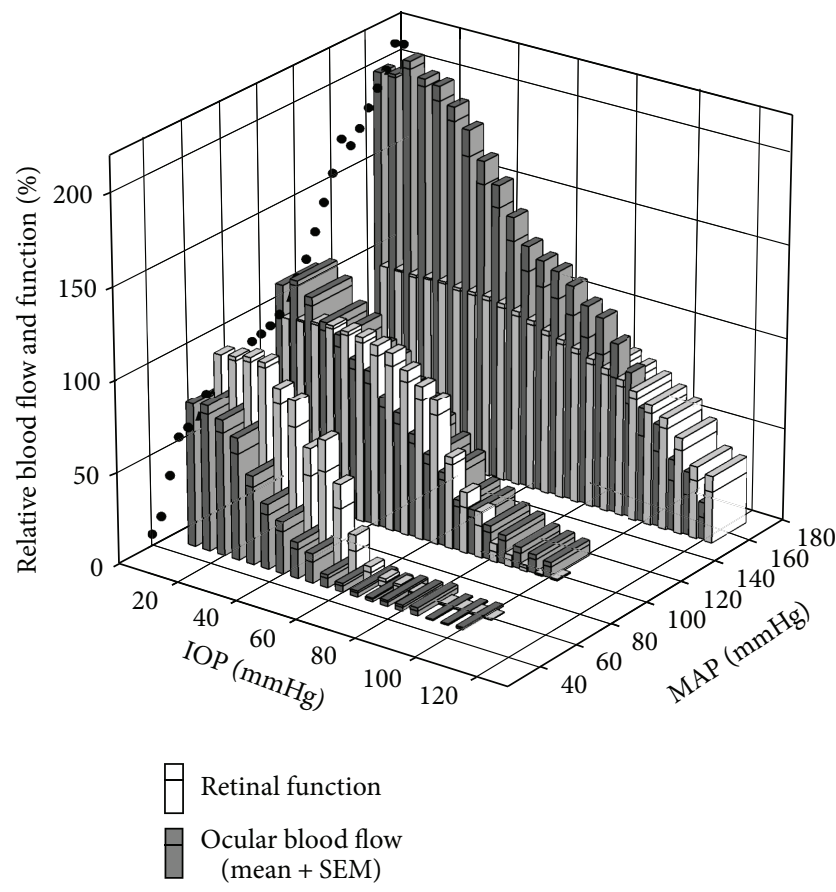

FIGURE 9: Comparison of relative retinal function and ocular blood flow during IOP elevation in animals with acute low, moderate, and high blood pressure. Black circles: blood flow autoregulation curve at baseline IOP. Function (unfilled bars, b-wave amplitude) is normalized to baseline. Starting blood flow (filled bars) is adjusted based on the autoregulation curve. Bars represent mean + SEM. Reprinted from [89] with permission.

\section{Other Factors Modifying the Response of the Rat ERG to IOP Elevation}

4.1. How Does Blood Pressure Influence the ERGs Response to IOP Elevation? As OPP expresses the balance between mean arterial pressure and IOP (OPP = MAP - IOP), a reduction in systemic blood pressure (BP) or a comparable increase in IOP should have similar effects on retinal function. For this reason there is increasing awareness that $\mathrm{BP}$ and thus OPP are important in glaucoma [90, 91]. Consistent with the above idea low BP has consistently been found to be a risk factor in glaucoma [92-99] and has shown to undermine normal blood-flow autoregulation within the primate optic nerve head [100]. At the other extreme, high BP has been reported to lower the risk of glaucoma [97, 99, 101, 102]. However, a host of other studies $[98,103-106]$ report that hypertension increases glaucoma risk.

Given the above, a better understanding of how IOP and $\mathrm{BP}$ interact to influence retinal function is needed. An acute experimental approach that affords accurate simultaneous control of both IOP and BP is useful as it avoids longterm cardiovascular confounds. This was recently achieved by measuring retinal function and blood flow over a wide range of OPPs, induced by IOP elevation in rats with low, moderate and high levels of acutely modified BP (Figure 9). In particular, pharmacological manipulation was employed to clamp BP at low and high BP levels for the duration of the IOP challenge. Figure 9 shows that in comparison to rats with normal BP, those with low and high BP show increased and decreased susceptibility to IOP elevation, respectively. This 
was true for both the b-wave amplitude and ocular blood flow. However, when function and blood flow are plotted as a function of OPP rather than IOP for the three BP groups, they do not overlie each other. Indeed, higher IOPs produced greater dysfunction even though the OPP was the same. It is likely that the additional mechanical load on the eye produced by the IOP elevation produces departures from the predicted OPP relationship due to mechanical effects. That is OPP should only be considered as a measure of vascular stress and may not be the metric that best describes glaucoma risk. The increased mechanical load leading to changes in the shape of the eye and optic nerve head may confer additional acute and chronic stresses on retinal ganglion cell axons and glia within the optic nerve head and in other peripapillary tissues.

4.2. Do Other Risk Factors Modify the ERGs Response to IOP Elevation? The above studies show that the ERG, in particular those components that arise from progressively more proximal retinal generators, are excellent measures of retinal stress when used in combination with a short IOP spike. This type of "stress test" is in some ways analogous to that used to assess the risk of cardiovascular disease. This concept has been applied in the clinic in an attempt to develop a more sensitive test of glaucoma. For example studies have measured the ERG during temporary IOP elevation using suction cup [107-110] or postural inversion [109, 111-113].

We adopt a similar approach in rodent models in order to assess how additional risk factors might influence the eye's response to IOP elevation. As discussed previously we find that the most sensitive metric of stress is the extent to which functional recovery is delayed and not the amount of functional loss. Indeed, repeated sampling during a protracted recovery allows the process to be quantified, thus reducing the variability associated with a single ERG measurement. Moreover, this approach using mild insult allows subtle differences in susceptibility to be revealed in a relatively rapid experimental paradigm [114]. As described below, we apply this approach to study the effect of aging on IOP susceptibility.

Advancing age is a major risk factor for glaucoma [115]. An emerging theory is that age-related mitochondrial dysfunction increases the vulnerability of neurons to injury [116]. We show that compared with 3-month-old C57 mice, older animals suffered greater decline in retinal function and slower recovery following IOP of $50 \mathrm{mmHg}$ for 30 minutes. This increased susceptibility is evident in 18-month-old mice. In the same study we were also able to show that alternate day fasting could ameliorate the increased susceptibility to the IOP stressor. This improvement in function was associated with increased mitochondrial enzyme expression and activity [117].

We undertook a parallel study in a murine model of accelerated aging. Polymerase $\gamma$ (PolG) $[118,119]$ is an enzyme that proofreads mitochondrial DNA as it is replicated [120]. Mice carrying systemic mutations in the proofreading domain of PolG $[121,122]$ exhibit premature senescence. We show that by 12 months of age PolG mice are more sensitive to IOP elevation [123]. These data provide support for the idea that mitochondrial dysfunction increases the susceptibility of the retina to IOP elevation.

\section{The ERG in Chronic IOP Elevation}

The response to a single mild IOP insult at best models the very earliest and reversible stages of injury that may ultimately contribute to ganglion cell loss in glaucoma. Not surprisingly chronic mild IOP elevation over the course of weeks produces a distinct pattern of ERG changes.

We have shown that chronic IOP elevation, induced by hypertonic saline injection into the episcleral veins in rats, produces a wide severity of retinal dysfunction [66]. The level of severity was best correlated with the mean IOP measured noninvasively four to five times a week over the six weeks of chronic IOP elevation. ERG deficits also showed a modest correlation with the severity of optic nerve damage assessed using gross histology.

Importantly we were able to show from data similar to that in Figure 10 that chronic IOP eyes could be grouped into those showing ERG dysfunction in the presence and absence of gross morphological change. These ERG deficits appeared to be greater for the pSTR (with some effect on the nSTR and the photopic b-wave) with little change to the a-wave or b-wave. In general, these animals had a mean IOP less than $30.2 \mathrm{mmHg}$. Eyes with clear signs of optic nerve changes tended to have higher mean IOP (mean $31.1 \mathrm{mmHg}$ ) and showed more severe ERG deficits, including almost complete loss of the pSTR, a marked reduction in nSTR, b-wave and scotopic and photopic OPs, with only a small effect on the ERG a-wave. Finally, in those animals showing higher IOP (mean $37.8 \mathrm{mmHg}$ ) and severe widespread optic nerve changes, the pSTR was completely abolished, and a- and bwaves, as well as photopic b-waves, were all reduced to less than $30 \%$ of control eye values.

5.1. How Do ERG Changes Differ in Acute and Chronic IOP Elevation? The above outcome suggests that selective RGC damage, as evident by attenuation of the STR, is produced by a relatively mild chronic elevation of IOP, whose mean over the six weeks of the experiment was less than $30 \mathrm{mmHg}$. This is consistent with the data for acute IOP elevation, where functional changes could be observed at an IOP of $30 \mathrm{mmHg}$. In particular, at $30 \mathrm{mmHg}$ a delay in the pSTR as well as in the trailing edge of the photopic ERG was evident, with little change to the bright flash a-wave or b-wave. Amplitude losses (nSTR) do not become apparent until IOP is elevated at least to $50 \mathrm{mmHg}$ (Figure 4 ).

The ERG deficits even at this low level of IOP elevation are associated with a mild decline in ocular blood flow, as shown in Figure 9 and more recently by Zhi et al. [51]. Thus it is likely that both mechanical and vascular stress is responsible for the dysfunction observed in both acute and chronic IOP elevation. What is likely is that the relative proportion of injury attributable to these mechanisms will differ in the two cases. Clearly dysfunction associated with acute IOP elevation lacks potential sources of stress arising from tissue 

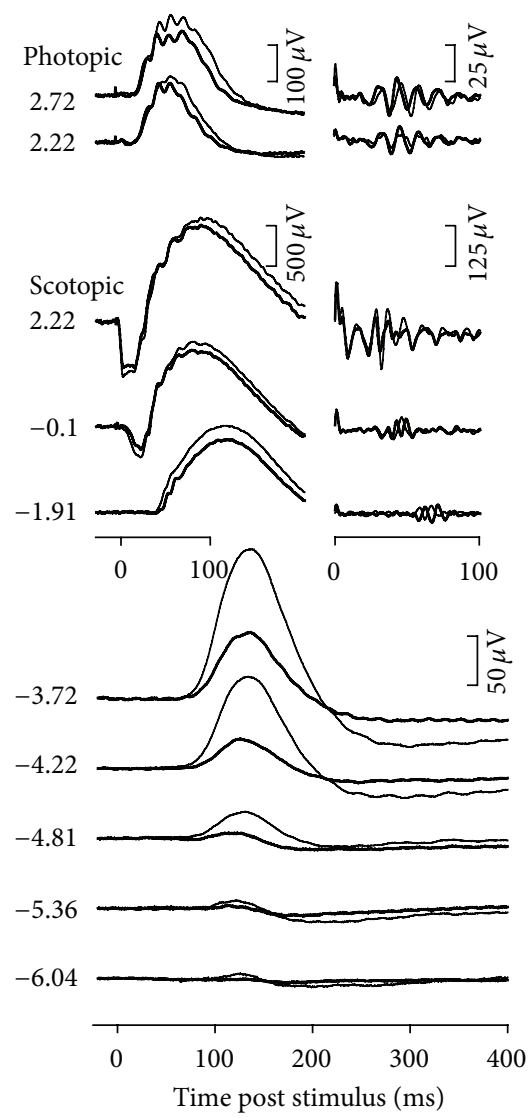

(a)
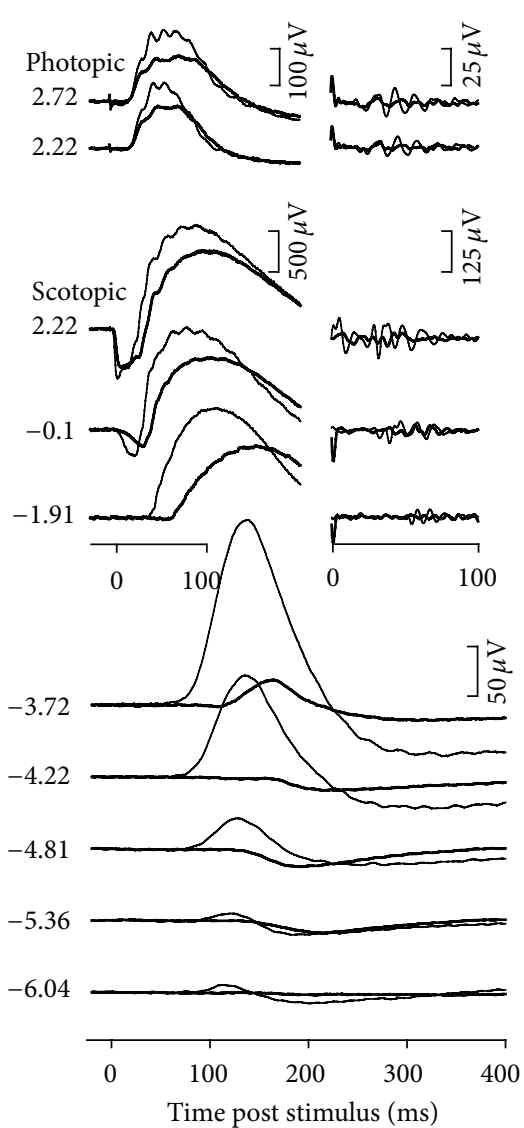

(b)
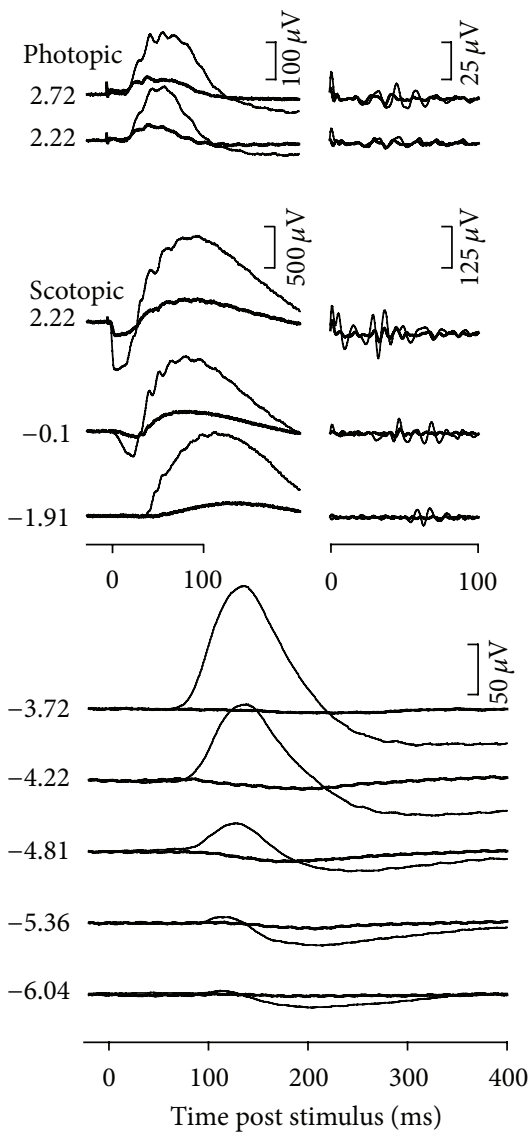

(c)

FIGURE 10: Effect of chronic IOP elevation on the rat ERG. Representative individual examples of ERG findings for experimental eyes (bold traces) and fellow control eyes (thin traces). Examples are given for eye showing mild (a), moderate (b), and high (c) IOP elevation. Stimulus flash intensities are listed at left for scotopic and photopic responses. Isolated OPs are shown to the right of corresponding waveforms. Reproduced with permission from [66].

remodelling of the optic nerve head associated with chronic IOP elevation.

A single IOP spike of $30 \mathrm{mmHg}$ is clearly not enough to cause permanent dysfunction as we have shown that the ERG completely recovers from short IOP spikes of $50 \mathrm{mmHg}$ and even $70 \mathrm{mmHg}$ (Figure 5). It is likely that chronic IOP elevation even at this mild level does not give the retina time to recover, thus leading to cumulative dysfunction. Figure 8(a) shows that repeated insults will progressively lengthen the time taken for recovery, particularly for the STR. One might extrapolate this to an extreme scenario whereby no recovery is possible. Figure 8(a) also demonstrates the idea that the accumulation of dysfunction will be more profound for the STR compared with the b-wave.

In general acute (Figure 4) and chronic IOP effects produce a similar pattern of loss to ERG components. That is, those components arising from the inner retina are more attenuated. An interesting difference between acute and chronic IOP effects is that the nSTR and the pSTR show greater sensitivity to the two conditions, respectively. In the acute case (Figure 4) the most sensitive component of the ERG is the negative component of the STR, whereas in the chronic situation the pSTR shows the largest deficit (Figure 10). The reasons for this are not clear. Our study employing optic nerve transection and tetrodotoxin application in rats suggests that the pSTR is almost entirely ganglion cell dependent in this species. On the other hand, the nSTR was not completely abolished following the same treatments suggesting that it contains "non-ganglion cell" or tetrodotoxininsensitive [14] contributions. One might speculate that such elements are sensitive to acute IOP elevation thus contributing to the greater attenuation of the nSTR during acute stress. Potential candidates include amacrine [124] and glial cells [125] both of which are known to be sensitive to acute hypoxic and hypoglycemic stress.

\section{Future Directions}

The above discussion shows that the magnitude, the duration, the frequency, and the recovery time between subsequent stressors and chronicity of IOP elevation all influence the functional integrity of the retina. The studies described above are by no means comprehensive and sample only a limited set of variables in a complex biological system. What remains 
to be defined is the exact IOP integral above which the retina becomes irreversibly damaged. Indeed, the idea of IOP integral as it relates to the point of irreversible damage needs to be revisited. Given our findings, it seems that IOP magnitude needs to be weighted more than the duration of stress. Moreover, it is likely that the weighting would need to be modified if IOP elevation were to occur in the setting of eyes with impaired autoregulation (e.g., diabetes, systemic hypertension, and vasospastic disease) or those prone to manifest more stress and strain at the optic nerve (e.g., altered biomechanics of optic nerve connective tissue in ageing, in myopia).

Clearly ERG dysfunction is related to some form of vascular stress. However, an explanation based purely on the vascular supply as expressed by OPP $=\mathrm{BP}-\mathrm{IOP}$ is inadequate to account for the spectrum of functional changes at various BPs. Indeed, a simple change in blood flow fails to explain ERG loss, as function seems to be robustly buffered against mild ischemia. There are clearly other regulatory mechanisms such as those involving oxygen extraction and local metabolic stores that need to be considered. What is needed is a study that simultaneously quantifies the effect of IOP elevation on the ERG, blood flow, and retinal oxygen tension. Such an approach would provide insights into the mechanisms that protect the retina from irreversible injury.

Whilst it is clear that acute changes in BP substantial modify the susceptibility of the ERG to IOP elevation (Figure 9), it is not known what effect chronic hypertension would have. The data of Figure 9, which are derived from acute manipulations, suggest that hypertension should be protective against elevated IOP. However, a number of epidemiological studies report that long-standing systemic hypertension increases the risk of glaucoma [98, 103-106]. Thus it will be important to assess the effect of IOP elevation in a model of chronic systemic hypertension. Moreover, the effect of chronic hypertension needs to be examined in a chronic model of glaucoma in order to provide greater clinical relevance.

\section{Summary}

In summary the ERG provides an excellent index of retinal stress. It is exquisitely sensitive to metabolic stress and IOP elevation and is useful for both acute and chronic studies in rodents. Employing ERG components arising from the inner retina along with a mild IOP elevation allows risk factors to be assessed in a controlled manner in rodent models of glaucoma. The development of a noninvasive means to elevate IOP in the rodent eye would allow for the application of a "stress test" to be employed in longitudinal assessment of preclinical disease models and give an indication of the effectiveness of their treatments.

\section{References}

[1] L. H. Cohen and W. K. Noell, "Glucose catabolism of rabbit retina before and after development of visual function," Journal of Neurochemistry, vol. 5, pp. 253-276, 1960.
[2] C. N. Graymore, "Metabolic survival of the isolated retina," British Medical Bulletin, vol. 26, no. 2, pp. 130-133, 1970.

[3] P. N. Dimitrov, B. N. Mukesh, C. A. McCarty, and H. R. Taylor, "Five-year incidence of bilateral cause-specific visual impairment in the Melbourne Visual Impairment Project," Investigative Ophthalmology and Visual Science, vol. 44, no. 12, pp. 5075-5081, 2003.

[4] H. A. Quigley, "Number of people with glaucoma worldwide," British Journal of Ophthalmology, vol. 80, no. 5, pp. 389-393, 1996.

[5] A. Sommer, "Intraocular pressure and glaucoma," American Journal of Ophthalmology, vol. 107, no. 2, pp. 186-188, 1989.

[6] M. O. Gordon, J. A. Beiser, J. D. Brandt et al., "The Ocular Hypertension Treatment Study: baseline factors that predict the onset of primary open-angle glaucoma," Archives of Ophthalmology, vol. 120, no. 6, pp. 714-730, 2002.

[7] M. A. Kass, D. K. Heuer, E. J. Higginbotham et al., "The Ocular Hypertension Treatment Study: a randomized trial determines that topical ocular hypotensive medication delays or prevents the onset of primary open-angle glaucoma," Archives of Ophthalmology, vol. 120, no. 6, pp. 701-730, 2002.

[8] "Origins of the electroretinogram," in Principles and Practice of Clinical Electrophysiology of Vision, J. R. Heckenlively and G. B. Arden, Eds., pp. 139-184, The MIT Press, Cambridge, Mass, USA, 2006.

[9] B. V. Bui, H. S. Weisinger, A. J. Sinclair, and A. J. Vingrys, "Comparison of guinea pig electroretinograms measured with bipolar corneal and unipolar intravitreal electrodes," Documenta Ophthalmologica, vol. 95, no. 1, pp. 15-34, 1998.

[10] B. Fortune, B. V. Bui, G. Cull, L. Wang, and G. A. Cioffi, "Interocular and inter-session reliability of the electroretinogram photopic negative response (PhNR) in non-human primates," Experimental Eye Research, vol. 78, no. 1, pp. 83-93, 2004.

[11] J. G. Robson and L. J. Frishman, "Dissecting the dark-adapted electroretinogram," Documenta Ophthalmologica, vol. 95, no. 34, pp. 187-215, 1998.

[12] T. M. Dang, T. I. Tsai, A. J. Vingrys, and B. V. Bui, "Postreceptoral contributions to the rat scotopic electroretinogram a-wave," Documenta Ophthalmologica, vol. 122, no. 3, pp. 149-156, 2011.

[13] F. Naarendorp, Y. Sato, A. Cajdric, and N. P. Hubbard, "Absolute and relative sensitivity of the scotopic system of rat: electroretinography and behavior," Visual Neuroscience, vol. 18, no. 4, pp. 641-656, 2001.

[14] B. V. Bui and B. Fortune, "Ganglion cell contributions to the rat full-field electroretinogram," Journal of Physiology, vol. 555, no. 1, pp. 153-173, 2004.

[15] S. M. Saszik, J. G. Robson, and L. J. Frishman, "The scotopic threshold response of the dark-adapted electroretinogram of the mouse," Journal of Physiology, vol. 543, no. 3, pp. 899-916, 2002.

[16] L. Wachtmeister, "Oscillatory potentials in the retina: what do they reveal," Progress in Retinal and Eye Research, vol. 17, no. 4, pp. 485-521, 1998.

[17] A. E. Weymouth and A. J. Vingrys, "Rodent electroretinography: methods for extraction and interpretation of rod and cone responses," Progress in Retinal and Eye Research, vol. 27, no. 1, pp. 1-44, 2008.

[18] J. J. Kang Derwent and R. A. Linsenmeier, "Intraretinal analysis of the a-wave of the electroretinogram (ERG) in dark-adapted intact cat retina," Visual Neuroscience, vol. 18, no. 3, pp. 353-363, 2001. 
[19] R. D. Braun, R. A. Linsenmeier, and T. K. Goldstick, "Oxygen consumption in the inner and outer retina of the cat," Investigative Ophthalmology and Visual Science, vol. 36, no. 3, pp. 542-554, 1995.

[20] D. R. Howard and D. C. Sawyer, "Electroretinography of acute hypoxic and increased intraocular pressure status in the dog," American Journal of Veterinary Research, vol. 36, no. 1, pp. 81-84, 1975.

[21] B. V. Bui, A. J. Vingrys, and M. Kalloniatis, "Correlating retinal function and amino acid immunocytochemistry following post-mortem ischemia," Experimental Eye Research, vol. 77, no. 2, pp. 125-136, 2003.

[22] P. L. Gehibach and R. L. Purple, "A paired comparison of two models of experimental retinal ischemia," Current Eye Research, vol. 13, no. 8, pp. 597-602, 1994.

[23] D. M. Rosenbaum, P. S. Rosenbaum, M. Singh et al., "Functional and morphologic comparison of two methods to produce transient retinal ischemia in the rat," Journal of NeuroOphthalmology, vol. 21, no. 1, pp. 62-68, 2001.

[24] B. V. Bui, M. Kalloniatis, and A. J. Vingrys, “The contribution of glycolytic and oxidative pathways to retinal photoreceptor function," Investigative Ophthalmology and Visual Science, vol. 44, no. 6, pp. 2708-2715, 2003.

[25] T. D. Lamb and E. N. Pugh, "A quantitative account of the activation steps involved in phototransduction in amphibian photoreceptors," Journal of Physiology, vol. 449, pp. 719-758, 1992.

[26] D. C. Hood and D. G. Birch, "Rod phototransduction in retinitis pigmentosa: estimation and interpretation of parameters derived from the rod a-wave," Investigative Ophthalmology and Visual Science, vol. 35, no. 7, pp. 2948-2961, 1994.

[27] J. Astrup, "Energy-requiring cell functions in the ischemic brain. Their critical supply and possible inhibition in protective therapy," Journal of Neurosurgery, vol. 56, no. 4, pp. 482-497, 1982.

[28] A. Ames, K. I. Maynard, and S. Kaplan, "Protection against CNS ischemia by temporary interruption of function- related processes of neurons," Journal of Cerebral Blood Flow and Metabolism, vol. 15, no. 3, pp. 433-439, 1995.

[29] S. J. Cringle, D.-Y. Yu, P. K. Yu, and E.-N. Su, "Intraretinal oxygen consumption in the rat in vivo," Investigative Ophthalmology and Visual Science, vol. 43, no. 6, pp. 1922-1927, 2002.

[30] L. Wang, M. Kondo, and A. Bill, "Glucose metabolism in cat outer retina: effects of light and hyperoxia," Investigative Ophthalmology and Visual Science, vol. 38, no. 1, pp. 48-55, 1997.

[31] R. A. Linsenmeier and C. M. Yancey, "Effects of hyperoxia on the oxygen distribution in the intact cat retina," Investigative Ophthalmology and Visual Science, vol. 30, no. 4, pp. 612-618, 1989.

[32] S. Viswanathan, L. J. Frishman, J. G. Robson, and J. W. Walters, "The photopic negative response of the flash electroretinogram in primary open angle glaucoma," Investigative Ophthalmology and Visual Science, vol. 42, no. 2, pp. 514-522, 2001.

[33] R. A. Bush and P. A. Sieving, "A proximal retinal component in the primate photopic ERG a-wave," Investigative Ophthalmology and Visual Science, vol. 35, no. 2, pp. 635-645, 1994.

[34] A. Szel and P. Rohlich, "Two cone types of rat retina detected by anti-visual pigment antibodies," Experimental Eye Research, vol. 55, no. 1, pp. 47-52, 1992.
[35] J. A. Jamison, R. A. Bush, B. Lei, and P. A. Sieving, "Characterization of the rod photoresponse isolated from the dark-adapted primate ERG," Visual Neuroscience, vol. 18, no. 3, pp. 445-455, 2001.

[36] C. Macaluso, S. Onoe, and G. Niemeyer, "Changes in glucose level affect rod function more than cone function in the isolated, perfused cat eye," Investigative Ophthalmology and Visual Science, vol. 33, no. 10, pp. 2798-2808, 1992.

[37] C. Hirsch-Hoffmann and G. Niemeyer, "Changes in plasma glucose level affect rod-, but not cone-ERG in the anesthetized cat," Clinical Vision Sciences, vol. 8, no. 6, pp. 489-501, 1993.

[38] M. Nihira, K. Anderson, F. A. Gorin, and M. S. Burns, "Primate rod and cone photoreceptors may differ in glucose accessibility," Investigative Ophthalmology and Visual Science, vol. 36, no. 7, pp. 1259-1270, 1995.

[39] A. Okubo, M. Sameshima, K. Unoki, F. Uehara, and N. Ohba, "Ultracytochemical demonstration of glycogen in cone, but not in rod, photoreceptor cells in the rat retina," Annals of Anatomy, vol. 180 , no. 4, pp. 307-314, 1998.

[40] T. R. Fricke, N. Mantzioros, and A. J. Vingrys, "Management of patients with narrow angles and acute angle-closure glaucoma," Clinical and Experimental Optometry, vol. 81, pp. 255-266, 1998.

[41] H. A. Quigley, "New paradigms in the mechanisms and management of glaucoma," Eye, vol. 19, no. 12, pp. 1241-1248, 2005.

[42] R. N. Weinreb and P. Tee Khaw, "Primary open-angle glaucoma," The Lancet, vol. 363, no. 9422, pp. 1711-1720, 2004.

[43] A. Sommer, J. M. Tielsch, J. Katz et al., "Relationship between intraocular pressure and primary open angle glaucoma among white and black Americans: the Baltimore eye survey," Archives of Ophthalmology, vol. 109, no. 8, pp. 1090-1095, 1991.

[44] A. Sommer, "Doyne lecture glaucoma: facts and fancies," Eye, vol. 10, part 3, pp. 295-301, 1996.

[45] S. Drange, D. R. Anderson, and M. Schulzer, "Risk factors for progression of visual field abnormalities in normal-tension glaucoma," American Journal of Ophthalmology, vol. 131, no. 6, pp. 699-708, 2001.

[46] B. V. Bui, B. Edmunds, G. A. Cioffi, and B. Fortune, "The gradient of retinal functional changes during acute intraocular pressure elevation," Investigative Ophthalmology and Visual Science, vol. 46, no. 1, pp. 202-213, 2005.

[47] W. S. Foulds and N. F. Johnson, "Rabbit electroretinogram during recovery from induced ischaemia," Transactions of the Ophthalmological Societies of the United Kingdom, vol. 94, no. 2, pp. 383-393, 1974.

[48] M. S. Marx, S. M. Podos, and I. Bodis-Wollner, "Flash and pattern electroretinograms in normal and laser-induced glaucomatous primate eyes," Investigative Ophthalmology and Visual Science, vol. 27, no. 3, pp. 378-386, 1986.

[49] R. Siliprandi, M. G. Bucci, R. Canella, and G. Carmignoto, "Flash and pattern electroretinograms during and after acute intraocular pressure elevation in cats," Investigative Ophthalmology and Visual Science, vol. 29, no. 4, pp. 558-565, 1988.

[50] J. G. Feghali, J. C. Jin, and J. V. Odom, "Effect of short-term intraocular pressure elevation on the rabbit electroretinogram," Investigative Ophthalmology and Visual Science, vol. 32, no. 8, pp. 2184-2189, 1991.

[51] Z. Zhi, W. Cepurna, E. Johnson, T. Shen, J. Morrison, and R. K. Wang, "Volumetric and quantitative imaging of retinal blood flow in rats with optical microangiography," Biomedical Optics Express, vol. 2, no. 3, pp. 579-591, 2011. 
[52] F. Block and M. Schwarz, "The b-wave of the electroretinogram as an index of retinal ischemia," General Pharmacology, vol. 30, no. 3, pp. 281-287, 1998.

[53] D. Sun, B. V. Bui, A. J. Vingrys, and M. Kalloniatis, "Alterations in photoreceptor-bipolar cell signaling following ischemia/reperfusion in the rat retina," Journal of Comparative Neurology, vol. 505, no. 1, pp. 131-146, 2007.

[54] D. G. Birch, D. C. Hood, S. Nusinowitz, and D. R. Pepperberg, "Abnormal activation and inactivation mechanisms of rod transduction in patients with autosomal dominant retinitis pigmentosa and the pro-23-his mutation," Investigative Ophthalmology and Visual Science, vol. 36, no. 8, pp. 1603-1614, 1995.

[55] D. R. Pepperberg, D. G. Birch, and D. C. Hood, "Photoresponses of human rods in vivo derived from paired-flash electroretinograms," Visual Neuroscience, vol. 14, no. 1, pp. 73-82, 1997.

[56] P. J. Nixon, B. V. Bui, J. A. Armitage, and A. J. Vingrys, "The contribution of cone responses to rat electroretinograms," Clinical and Experimental Ophthalmology, vol. 29, no. 3, pp. 193-196, 2001.

[57] Z. He, B. V. Bui, and A. J. Vingrys, "The rate of functional recovery from acute IOP elevation," Investigative Ophthalmology and Visual Science, vol. 47, no. 11, pp. 4872-4880, 2006.

[58] M. D. Roberts, Y. Liang, I. A. Sigal et al., "Correlation between local stress and strain and lamina cribrosa connective tissue volume fraction in normal monkey eyes," Investigative Ophthalmology and Visual Science, vol. 51, no. 1, pp. 295-307, 2010.

[59] I. A. Sigal, H. Yang, M. D. Roberts, C. F. Burgoyne, and J. Crawford Downs, "IOP-induced lamina cribrosa displacement and scleral canal expansion: an analysis of factor interactions using parameterized eye-specific models," Investigative Ophthalmology and Visual Science, vol. 52, no. 3, pp. 9023-9032, 2011.

[60] B. Fortune, T. E. Choe, J. Reynaud et al., "Deformation of the rodent optic nerve head and peripapillary structures during acute intraocular pressure elevation," Investigative Ophthalmology and Visual Science, vol. 52, no. 9, pp. 6651-6661, 2011.

[61] S. J. McKinnon, “Glaucoma: ocular Alzheimer's disease?” Frontiers in Bioscience, vol. 8, pp. s1140-s1156, 2003.

[62] F. Mabuchi, M. Aihara, M. R. Mackey, J. D. Lindsey, and R. N. Weinreb, "Optic nerve damage in experimental mouse ocular hypertension," Investigative Ophthalmology and Visual Science, vol. 44, no. 10, pp. 4321-4330, 2003.

[63] L. Guo, S. E. Moss, R. A. Alexander, R. R. Ali, F. W. Fitzke, and M. F. Cordeiro, "Retinal ganglion cell apoptosis in glaucoma is related to intraocular pressure and IOP-induced effects on extracellular matrix," Investigative Ophthalmology and Visual Science, vol. 46, no. 1, pp. 175-182, 2005.

[64] B. C. Chauhan, J. Pan, M. L. Archibald, T. L. LeVatte, M. E. M. Kelly, and F. Tremblay, "Effect of intraocular pressure on optic disc topography, electroretinography, and axonal loss in a chronic pressure-induced rat model of optic nerve damage," Investigative Ophthalmology and Visual Science, vol. 43, no. 9, pp. 2969-2976, 2002.

[65] W. A. Hare, E. WoldeMussie, R. K. Lai et al., "Efficacy and safety of memantine treatment for reduction of changes associated with experimental glaucoma in monkey, I: functional measures," Investigative Ophthalmology and Visual Science, vol. 45, no. 8, pp. 2625-2639, 2004.
[66] B. Fortune, B. V. Bui, J. C. Morrison et al., "Selective ganglion cell functional loss in rats with experimental glaucoma," Investigative Ophthalmology and Visual Science, vol. 45, no. 6, pp. 1854-1862, 2004.

[67] S. M. Drance, "Diurnal variation of intraocular pressure in treated glaucoma. Significance in patients with chronic simple glaucoma," Archives of Ophthalmology, vol. 70, pp. 302-311, 1963.

[68] S. M. DRANCE, "The significance of the diurnal tension variations in normal and glaucomatous eyes," Archives of Ophthalmology, vol. 64, pp. 494-501, 1960.

[69] S. Mosaed, J. H. K. Liu, and R. N. Weinreb, "Correlation between office and peak nocturnal intraocular pressures in healthy subjects and glaucoma patients," American Journal of Ophthalmology, vol. 139, no. 2, pp. 320-324, 2005.

[70] J. H. K. Liu, X. Zhang, D. F. Kripke, and R. N. Weinreb, “Twentyfour-hour intraocular pressure pattern associated with early glaucomatous changes," Investigative Ophthalmology and Visual Science, vol. 44, no. 4, pp. 1586-1590, 2003.

[71] J. W. McLaren, R. F. Brubaker, and J. S. Fitzsimon, "Continuous measurement of intraocular pressure in rabbits by telemetry," Investigative Ophthalmology and Visual Science, vol. 37, no. 6, pp. 966-975, 1996.

[72] C. G. Moore, E. C. Johnson, and J. C. Morrison, "Circadian rhythm of intraocular pressure in the rat," Current Eye Research, vol. 15, no. 2, pp. 185-191, 1996.

[73] M. Aihara, J. D. Lindsey, and R. N. Weinreb, “Twenty-fourhour pattern of mouse intraocular pressure," Experimental Eye Research, vol. 77, no. 6, pp. 681-686, 2003.

[74] S. C. Saccà, M. Rolando, A. Marletta, A. Macri, P. Cerqueti, and G. Ciurlo, "Fluctuations of intraocular pressure during the day in open-angle glaucoma, normal-tension glaucoma and normal subjects," Ophthalmologica, vol. 212, no. 2, pp. 115-119, 1998.

[75] K. Nouri-Mahdavi, D. Hoffman, A. L. Coleman et al., "Predictive factors for glaucomatous visual field progression in the Advanced Glaucoma Intervention Study," Ophthalmology, vol. 111, no. 9, pp. 1627-1635, 2004.

[76] S. Asrani, R. Zeimer, J. Wilensky, D. Gieser, S. Vitale, and K. Lindenmuth, "Large diurnal fluctuations in intraocular pressure are an independent risk factor in patients with glaucoma," Journal of Glaucoma, vol. 9, no. 2, pp. 134-142, 2000.

[77] B. Bengtsson, M. C. Leske, L. Hyman, and A. Heijl, "Fluctuation of intraocular pressure and glaucoma progression in the early manifest glaucoma trial," Ophthalmology, vol. 114, no. 2, pp. 205-209, 2007.

[78] Z. He, B. V. Bui, and A. J. Vingrys, "Effect of repeated IOP challenge on rat retinal function," Investigative Ophthalmology and Visual Science, vol. 49, no. 7, pp. 3026-3034, 2008.

[79] R. A. Linsenmeier, A. H. Mines, and R. H. Steinberg, "Effects of hypoxia and hypercapnia on the light peak and electroretinogram of the cat," Investigative Ophthalmology and Visual Science, vol. 24, no. 1, pp. 37-46, 1983.

[80] C. M. Yancey and R. A. Linsenmeier, "The electroretinogram and choroidal $\mathrm{PO}_{2}$ in the cat during elevated intraocular pressure," Investigative Ophthalmology and Visual Science, vol. 29, no. 5, pp. 700-707, 1988.

[81] C. M. Yancey and R. A. Linsenmeier, "Oxygen distribution and consumption in the cat retina at increased intraocular pressure," Investigative Ophthalmology and Visual Science, vol. 30, no. 4, pp. 600-611, 1989. 
[82] E. Stefánsson, D. B. Pedersen, P. K. Jensen et al., "Optic nerve oxygenation," Progress in Retinal and Eye Research, vol. 24, no. 3, pp. 307-332, 2005.

[83] D. J. Brooks, "The clinical role of PET in cerebrovascular disease," Neurosurgical Review, vol. 14, no. 2, pp. 91-96, 1991.

[84] D. R. Dantzker, "Oxygen delivery and utilization," Applied Cardiopulmonary Pathophysiology, vol. 3, no. 4, pp. 345-350, 1991.

[85] L. Friberg, J. Olesen, N. A. Lassen, T. S. Olsen, and A. Karle, "Cerebral oxygen extraction, oxygen consumption, and regional cerebral blood flow during the aura phase of migraine," Stroke, vol. 25, no. 5, pp. 974-979, 1994.

[86] C. K. Chapler and S. M. Cain, "The physiologic reserve in oxygen carrying capacity: studies in experimental hemodilution," Canadian Journal of Physiology and Pharmacology, vol. 64, no. 1, pp. 7-12, 1986.

[87] J. R. Sutton, "Limitations to maximal oxygen uptake," Sports Medicine, vol. 13, no. 2, pp. 127-133, 1992.

[88] P. Tornquist and A. Alm, "Retinal and choroidal contribution to retinal metabolism in vivo. A study in pigs," Acta Physiologica Scandinavica, vol. 106, no. 3, pp. 351-357, 1979.

[89] Z. He, C. T. O. Nguyen, J. A. Armitage, A. J. Vingrys, and B. V. Bui, "Blood pressure modifies retinal susceptibility to intraocular pressure elevation," PLoS ONE, vol. 7, no. 2, Article ID e31104, 2012.

[90] J. Caprioli and A. L. Coleman, "Blood pressure, perfusion pressure, and glaucoma," American Journal of Ophthalmology, vol. 149, no. 5, pp. 704-712, 2010.

[91] J. M. Tielsch, J. Katz, A. Sommer, H. A. Quigley, and J. C. Javitt, "Hypertension, perfusion pressure, and primary openangle glaucoma: a population-based assessment," Archives of Ophthalmology, vol. 113, no. 2, pp. 216-221, 1995.

[92] N. Collignon, W. Dewe, S. Guillaume, and J. Collignon-Brach, "Ambulatory blood pressure monitoring in glaucoma patients. The nocturnal systolic dip and its relationship with disease progression," International Ophthalmology, vol. 22, no. 1, pp. 19-25, 1998.

[93] P. Demailly, F. Cambien, and P. F. Plouin, "Do patients with low tension glaucoma have particular cardiovascular characteristics?” Ophthalmologica, vol. 188, no. 2, pp. 65-75, 1984.

[94] S. L. Graham and S. M. Drance, "Nocturnal hypotension: role in glaucoma progression," Survey of Ophthalmology, vol. 43, no. 6, pp. S10-S16, 1999.

[95] S. L. Graham, S. M. Drance, K. Wijsman, G. R. Douglas, and F. S. Mikelberg, "Ambulatory blood pressure monitoring in glaucoma: the nocturnal dip," Ophthalmology, vol. 102, no. 1, pp. 61-69, 1995.

[96] H. J. Kaiser, J. Flammer, T. Graf, and D. Stumpfig, "Systemic blood pressure in glaucoma patients," Graefe's Archive for Clinical and Experimental Ophthalmology, vol. 231, no. 12, pp. 677-680, 1993.

[97] M. C. Leske, A. Heijl, L. Hyman, B. Bengtsson, L. Dong, and Z. Yang, "Predictors of long-term progression in the early manifest glaucoma trial," Ophthalmology, vol. 114, no. 11, pp. 1965-1972, 2007.

[98] F. Memarzadeh, M. Ying-Lai, J. Chung, S. P. Azen, and R. Varma, "Blood pressure, perfusion pressure, and open-angle glaucoma: the Los Angeles Latino eye study," Investigative Ophthalmology and Visual Science, vol. 51, no. 6, pp. 2872-2877, 2010.

[99] F. Topouzis, A. L. Coleman, A. Harris et al., "Association of blood pressure status with the optic disk structure in non-glaucoma subjects: the Thessaloniki eye study," American Journal of Ophthalmology, vol. 142, no. 1, pp. 60-67.e1, 2006.

[100] Y. Liang, J. C. Downs, B. Fortune, G. Cull, G. A. Cioffi, and L. Wang, "Impact of systemic blood pressure on the relationship between intraocular pressure and blood flow in the optic nerve head of nonhuman primates," Investigative Ophthalmology and Visual Science, vol. 50, no. 5, pp. 2154-2160, 2009.

[101] M. C. Leske, S. Y. Wu, A. Hennis, R. Honkanen, and B. Nemesure, "Risk factors for incident open-angle glaucoma: the Barbados Eye Studies," Ophthalmology, vol. 115, no. 1, pp. 85-93, 2008.

[102] M. C. Leske, S. Y. Wu, B. Nemesure, and A. Hennis, "Incident open-angle glaucoma and blood pressure," Archives of Ophthalmology, vol. 120, no. 7, pp. 954-959, 2002.

[103] L. Bonomi, G. Marchini, M. Marraffa, P. Bernardi, R. Morbio, and A. Varotto, "Vascular risk factors for primary open angle glaucoma: the Egna-Neumarkt Study," Ophthalmology, vol. 107, no. 7, pp. 1287-1293, 2000.

[104] I. Dielemans, J. R. Vingerling, D. Algra, A. Hofman, D. E. Grobbee, and P. T. V. M. De Jong, "Primary open-angle glaucoma, intraocular pressure, and systemic blood pressure in the general elderly population: the Rotterdam Study," Ophthalmology, vol. 102, no. 1, pp. 54-60, 1995.

[105] C. A. A. Hulsman, J. R. Vingerling, A. Hofman, J. C. M. Witteman, and P. T. V. M. De Jong, "Blood pressure, arterial stiffness, and open-angle glaucoma: the Rotterdam Study," Archives of Ophthalmology, vol. 125, no. 6, pp. 805-812, 2007.

[106] P. Mitchell, A. J. Lee, E. Rochtchina, and J. J. Wang, "Open-angle glaucoma and systemic hypertension: the Blue Mountains Eye Study," Journal of Glaucoma, vol. 13, no. 4, pp. 319-326, 2004.

[107] A. Azuara-Blanco, A. Harris, L. B. Cantor, M. M. Abreu, and M. Weinland, "Effects of short term increase of intraocular pressure on optic disc cupping," British Journal of Ophthalmology, vol. 82, no. 8, pp. 880-883, 1998.

[108] A. Colotto, B. Falsini, T. Salgarello, L. Buzzonetti, S. Cermola, and G. Porrello, "Transiently raised intraocular pressure reveals pattern electroretinogram losses in ocular hypertension," Investigative Ophthalmology and Visual Science, vol. 37, no. 13, pp. 2663-2670, 1996.

[109] T. R. Friberg and G. Sanborn, "Optic nerve dysfunction during gravity inversion. Pattern reversal visual evoked potentials," Archives of Ophthalmology, vol. 103, no. 11, pp. 1687-1689, 1985.

[110] J. V. Lovasik, H. Kergoat, and M. Gagnon, "Experimentally reduced perfusion of one eye impairs retinal function in both eyes," Optometry and Vision Science, vol. 82, no. 9, pp. 850-857, 2005.

[111] M. Nagaraju, M. Saleh, and V. Porciatti, "IOP-dependent retinal ganglion cell dysfunction in glaucomatous DBA/2J mice," Investigative Ophthalmology and Visual Science, vol. 48, no. 10, pp. 4573-4579, 2007.

[112] L. M. Ventura, I. Golubev, W. Lee, I. Nose, J. M. Parel et al., "Head-down posture induces PERG alterations in early glaucoma," Journal of Glaucoma. In press.

[113] G. Ferreri, R. Buceti, F. M. B. Ferreri, and A. M. Roszkowska, "Postural modifications of the oscillatory potentials of the electroretinogram in primary open-angle glaucoma," Ophthalmologica, vol. 216, no. 1, pp. 22-26, 2002.

[114] Y. X. Kong, J. G. Crowston, A. J. Vingrys, I. A. Trounce, and B. V. Bui, "Functional changes in the retina during and after acute intraocular pressure elevation in mice," Investigative Ophthalmology and Visual Science, vol. 50, no. 12, pp. 5732-5740, 2009. 
[115] H. A. Quigley and A. T. Broman, "The number of people with glaucoma worldwide in 2010 and 2020," British Journal of Ophthalmology, vol. 90, no. 3, pp. 262-267, 2006.

[116] M. P. Mattson and T. Magnus, "Ageing and neuronal vulnerability," Nature Reviews Neuroscience, vol. 7, no. 4, pp. 278-294, 2006.

[117] Y. X. G. Kong, N. van Bergen, B. V. Bui et al., "Impact of aging and diet restriction on retinal function during and after acute intraocular pressure injury," Neurobiology of Aging, vol. 33, no. 6, pp. 1126e15-1126e25, 2012.

[118] L. S. Kaguni, "DNA polymerase $\gamma$, the mitochondrial replicase," Annual Review of Biochemistry, vol. 73, pp. 293-320, 2004.

[119] M. A. Graziewicz, M. J. Longley, and W. C. Copeland, "DNA polymerase $\gamma$ in mitochondrial DNA replication and repair," Chemical Reviews, vol. 106, no. 2, pp. 383-405, 2006.

[120] M. J. Longley, D. Nguyen, T. A. Kunkel, and W. C. Copeland, "The fidelity of human DNA polymerase gamma with and without exonucleolytic proofreading and the p55 accessory subunit," The Journal of Biological Chemistry, vol. 276, pp. 38555-38562, 2001.

[121] C. C. Kujoth, A. Hiona, T. D. Pugh et al., "Medicine: mitochondrial DNA mutations, oxidative stress, and apoptosis in mammalian aging," Science, vol. 309, no. 5733, pp. 481-484, 2005.

[122] A. Trifunovic, A. Hansson, A. Wredenberg et al., "Somatic mtDNA mutations cause aging phenotypes without affecting reactive oxygen species production," Proceedings of the National Academy of Sciences of the United States of America, vol. 102, no. 50, pp. 17993-17998, 2005.

[123] Y. X. G. Kong, N. van Bergen, I. A. Trounce et al., "Increase in mitochondrial DNA mutations impairs retinal function and renders the retina vulnerable to injury," Aging Cell, vol. 10, no. 4, pp. 572-583, 2011.

[124] X. Luo, G. N. Lambrou, J. A. Sahel, and D. Hicks, "Hypoglycemia induces general neuronal death, whereas hypoxia and glutamate transport blockade lead to selective retinal ganglion cell death in vitro," Investigative Ophthalmology and Visual Science, vol. 42, no. 11, pp. 2695-2705, 2001.

[125] T. T. Lam, J. M. K. Kwong, and M. O. M. Tso, "Early glial responses after acute elevated intraocular pressure in rats," Investigative Ophthalmology and Visual Science, vol. 44, no. 2, pp. 638-645, 2003. 


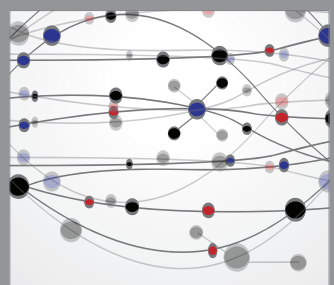

The Scientific World Journal
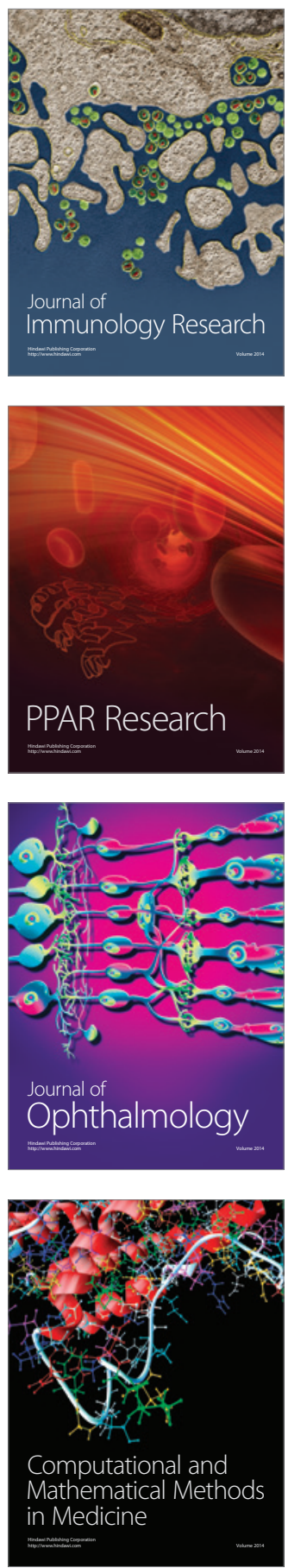

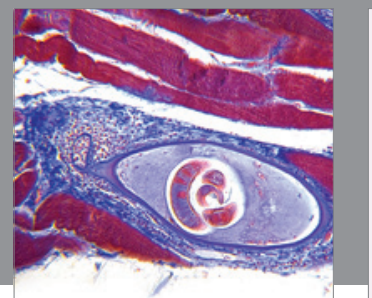

Gastroenterology

Research and Practice
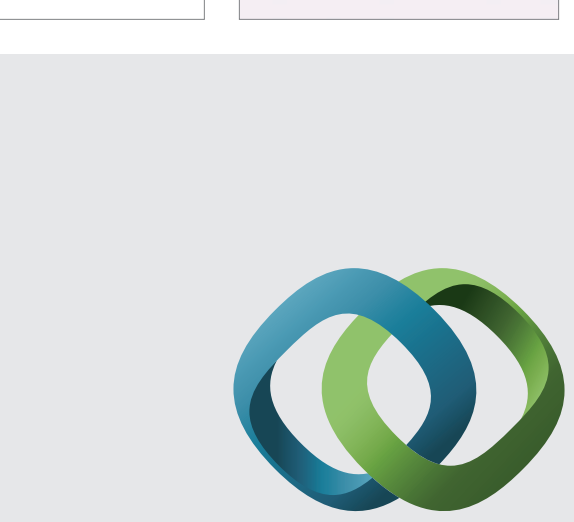

\section{Hindawi}

Submit your manuscripts at

http://www.hindawi.com
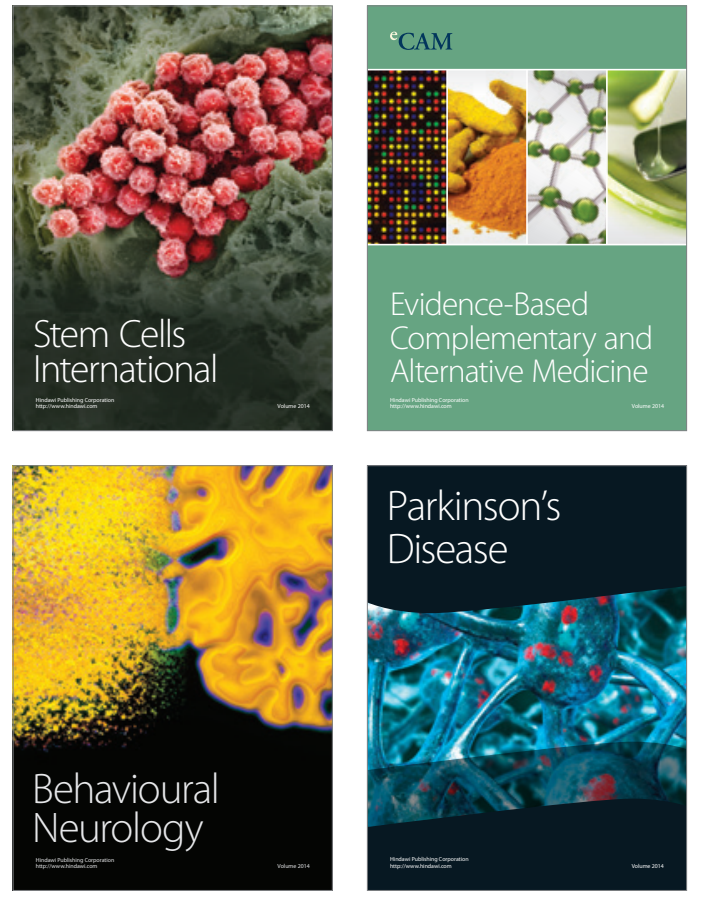
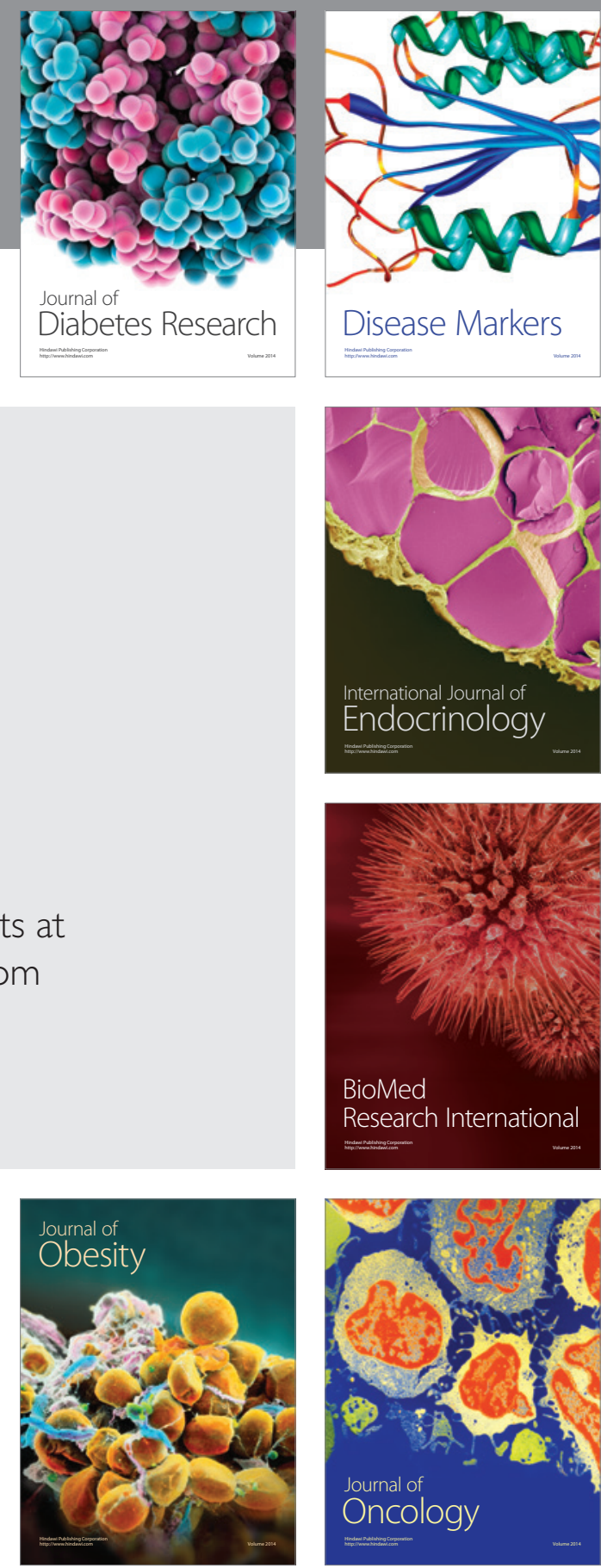

Disease Markers
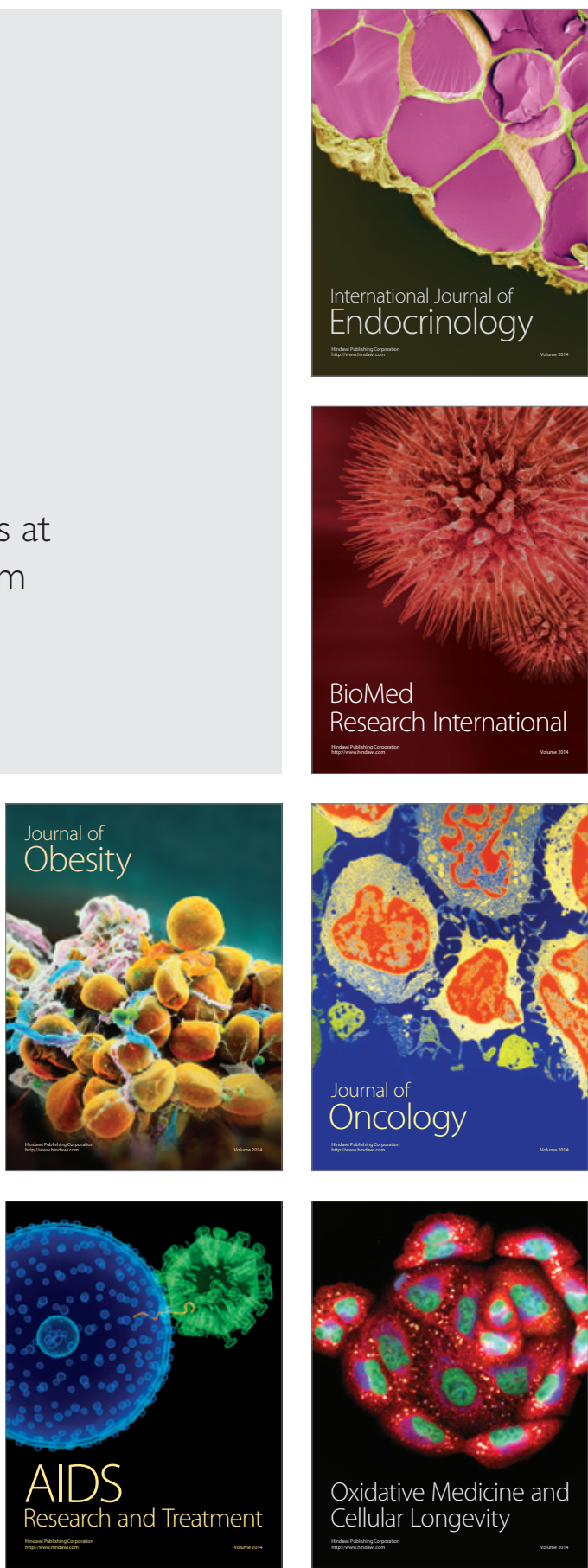